\title{
INTERPOLATION OF INTERSECTIONS BY THE REAL METHOD
}

\author{
S. V. ASTASHKIN
}

\begin{abstract}
Let $\left(X_{0}, X_{1}\right)$ be a Banach couple with $X_{0} \cap X_{1}$ dense both in $X_{0}$ and in $X_{1}$, and let $\left(X_{0}, X_{1}\right)_{\theta, q}(0<\theta<1,1 \leq q<\infty)$ denote the real interpolation spaces. Suppose $\psi$ is a linear functional defined on some linear subspace $M \subset X_{0}+X_{1}$ and satisfying $\psi \in\left(X_{0} \cap X_{1}\right)^{*}, \psi \neq 0$. Conditions are considered that ensure the natural identity

$$
\left(X_{0} \cap \operatorname{Ker} \psi, X_{1} \cap \operatorname{Ker} \psi\right)_{\theta, q}=\left(X_{0}, X_{1}\right)_{\theta, q} \cap \operatorname{Ker} \psi .
$$

The results obtained provide a solution for the problem posed by N. Krugljak, L. Maligranda, and L.-E. Persson and pertaining to interpolation of couples of intersections that are generated by an integral functional in a weighted $L_{p}$-scale. Furthermore, an expression is found for the $\mathcal{K}$-functional on a couple of intersections corresponding to a linear functional, and some other related questions are treated.
\end{abstract}

\section{INTRODUCTION}

Let $\left(X_{0}, X_{1}\right)$ be a normed couple, i.e., a couple of normed spaces embedded linearly and continuously in a Hausdorff linear topological space $\mathcal{T}$. For $x \in X_{0}+X_{1}$ and $t>0$, we define the Peetre $\mathcal{K}$-functional

$$
\mathcal{K}\left(t, x ; X_{0}, X_{1}\right)=\inf \left\{\left\|x_{0}\right\|_{X_{0}}+t\left\|x_{1}\right\|_{X_{1}}: x=x_{0}+x_{1}, x_{0} \in X_{0}, x_{1} \in X_{1}\right\} .
$$

If $0<\theta<1$ and $1 \leq q<\infty$, the real interpolation space $X_{\theta, q}=\left(X_{0}, X_{1}\right)_{\theta, q}$ consists of all $x \in X_{0}+X_{1}$ with

$$
\|x\|_{\theta, q}=\left\{\int_{0}^{\infty}\left(t^{-\theta} \mathcal{K}\left(t, x: X_{0}, X_{1}\right)\right)^{q} \frac{d t}{t}\right\}^{1 / q}<\infty .
$$

Next, if $\left(X_{0}, X_{1}\right)$ is a Banach couple, then each linear subspace $N \subset \mathcal{T}$ determines the normed (in general, non-Banach) couple $\left(X_{0} \cap N, X_{1} \cap N\right)$ of intersections. The norm on $X_{i} \cap N$ is simply the restriction of the norm of $X_{i}(i=0,1)$.

Now, it is natural to look for conditions ensuring the natural identity

$$
\left(X_{0} \cap N, X_{1} \cap N\right)_{\theta, q}=\left(X_{0}, X_{1}\right)_{\theta, q} \cap N
$$

(with equivalence of the norms).

This formula is true if, for instance, $\left(X_{0}, X_{1}\right)$ is a couple of Banach function lattices and $N$ is a linear space of functions (on the same measure space) with the lattice property: if $g \in N$ and $|f| \leq|g|$, then $f \in N$ (see [1, Remark 2]). On the other hand, if we take the couple $\left(L_{1}[0,1], L_{\infty}[0,1]\right)$ for $\left(X_{0}, X_{1}\right)$ and the closed linear span $\left[r_{n}\right]$ of the Rademacher system in $L_{1}[0,1]$ for $N$, then $(0.1)$ fails. Indeed, by the Khinchin inequality, $L_{p} \cap\left[r_{n}\right] \approx l_{2}$ for $1 \leq p<\infty$, and $L_{\infty} \cap\left[r_{n}\right] \approx l_{1}$. Thus, calculating the interpolation spaces in (0.1) for arbitrary $0<\theta<1$ and $q=\frac{2}{1+\theta}$ (see [2, Theorem 5.2.1]), we arrive at $\left(X_{0} \cap N, X_{1} \cap N\right)_{\theta, q} \approx l_{q}$, and $\left(X_{0}, X_{1}\right)_{\theta, q} \cap N \approx l_{2}$.

2000 Mathematics Subject Classification. Primary 46B70.

Key words and phrases. Interpolation space, real interpolation method, $\mathcal{K}$-functional, dilation indices of a function, spaces of measurable functions, weighted spaces. 
In this paper we are interested in the case where $N$ is the kernel of a linear functional defined on some linear subspace $D \subset \mathcal{T}$. An important particular case of this problem was treated in [1]. Namely, in that paper certain conditions on $\theta \in(0,1), p \in[1, \infty)$, and on weight functions $w_{0}(x)$ and $w_{1}(x)$ were found, ensuring the formula

$$
\left(L_{p}\left(w_{0}\right) \cap N, L_{p}\left(w_{1}\right) \cap N\right)_{\theta, p}=\left(L_{p}\left(w_{0}\right), L_{p}\left(w_{1}\right)\right)_{\theta, p} \cap N,
$$

where $L_{p}(w)$ is the weighted $L_{p}$-space on $(0, \infty)$ with the usual norm, and $N$ is the space of all functions $f:(0, \infty) \rightarrow \mathbb{R}$ satisfying

$$
\int_{0}^{\infty} f(x) d x=0 .
$$

It turns out that the validity of $(0.2)$ is closely related to the possibility of "interpolating" certain Hardy-type integral inequalities. In the same paper [1], the following more general question was asked: Under what conditions on $w_{0}(x), w_{1}(x), p_{0}, p_{1} \in[1, \infty), \theta \in(0,1)$, and $q \in[1, \infty]$ do we have the identity

$$
\left(L_{p_{0}}\left(w_{0}\right) \cap N, L_{p_{1}}\left(w_{1}\right) \cap N\right)_{\theta, q}=\left(L_{p_{0}}\left(w_{0}\right), L_{p_{1}}\left(w_{1}\right)\right)_{\theta, q} \cap N ?
$$

(Generally speaking, here $p_{0} \neq p_{1}$, but $N$ is still determined by (3).)

It is well known (see [2, Chapter 5]) that the standard equivalent expressions for the $\mathcal{K}$-functional on the couple $\left(L_{p_{0}}\left(w_{0}\right), L_{p_{1}}\left(w_{1}\right)\right)$ differ substantially in the cases of $p_{0}=p_{1}$ and of $p_{0} \neq p_{1}$; as a result, the latter question is highly more complicated than that treated in [1]. This was the origin for the following general problem.

Suppose $\left(X_{0}, X_{1}\right)$ is a Banach couple with $X_{i} \subset \mathcal{T}(i=0,1)$ and such that $X_{0} \cap X_{1}$ is dense both in $X_{0}$ and in $X_{1}$. Next, let $\psi$ be a linear functional defined on some linear space $D \subset \mathcal{T}$, let $\psi$ belong to $\left(X_{0} \cap X_{1}\right)^{*}$, and let $\psi(x) \neq 0$ for some $x \in X_{0} \cap X_{1}$. We shall see that, for the validity of the formula

$$
\left(X_{0} \cap \operatorname{Ker} \psi, X_{1} \cap \operatorname{Ker} \psi\right)_{\theta, q}=\left(X_{0}, X_{1}\right)_{\theta, q} \cap \operatorname{Ker} \psi,
$$

of primary importance are four indices, $\alpha, \beta, \alpha_{0}$, and $\beta_{\infty}$, related to the Peetre $\mathcal{K}$ functional $\mathcal{K}\left(t, \psi ; X_{0}^{*}, X_{1}^{*}\right)$ in the couple $\left(X_{0}^{*}, X_{1}^{*}\right)$ of dual spaces. These indices satisfy the inequalities

$$
0 \leq \alpha \leq \min \left(\alpha_{0}, \beta_{\infty}\right) \leq \max \left(\alpha_{0}, \beta_{\infty}\right) \leq \beta \leq 1
$$

It will be shown that, under the additional condition $\beta_{\infty} \leq \alpha_{0}$, the norms of the interpolation spaces $X_{\theta, q}=\left(X_{0}, X_{1}\right)_{\theta, q}$ and $N_{\theta, q}=\left(N_{0}, N_{1}\right)_{\theta, q}$ are equivalent on $N:=$ $X_{0} \cap X_{1} \cap \operatorname{Ker} \psi$ if and only if $\theta \in(0, \alpha) \cup\left(\beta_{\infty}, \alpha_{0}\right) \cup(\beta, 1)$ (see Theorem 1$)$. Here $N_{i}$ is the space $N$ endowed with the norm of $X_{i}(i=0,1)$. So, the latter condition is necessary for (0.5) to be true. Furthermore, we shall show that if $\psi$ is defined on $X_{0} \cap X_{1}$, $\psi \in\left(X_{0} \cap X_{1}\right)^{*}$, then for every $\theta \in(0, \alpha) \cup\left(\beta_{\infty}, \alpha_{0}\right) \cup(\beta, 1)$ the functional $\psi$ admits an extension by continuity to some space $\widetilde{M}_{\theta} \supset X_{0} \cap X_{1}$ such that $(0.5)$ is fulfilled for the kernel of this extension (see Theorem 2).

In the proof of Theorem 1, an approach developed by Ivanov and Kalton in [3] is used. The first part of [3] was devoted to comparison of the interpolation spaces $\left(X_{0}, X_{1}\right)_{\theta, q}$ and $\left(Y_{0}, X_{1}\right)_{\theta, q}(0<\theta<1,1 \leq q<\infty)$, where $Y_{0}=\operatorname{Ker} \psi, \psi \in X_{0}^{*}$. The essence of that approach is in the reduction of the general interpolation problem for subspaces to the study of the shift operator in a certain weighted $l_{p}$-space. At the same time, the results of [3] are consequences of those obtained here (see Corollary 3 and Remark 2).

It should be noted that interpolation of intersections and the problem studied in [3] are only partial (though important) cases of the general subspace interpolation problem formulated as early as in the monograph [4 by Lions and Magenes. Various aspects of this general problem have been treated in [5]-[14] (the author is very far from claiming the completeness of this citation list). 
Under certain conditions, the results of the first part of the paper allow us to answer the question from [1] mentioned above. In this setting, $N$ is the kernel of the integral functional $\psi(f)=\int_{0}^{\infty} f(x) d x$. Not entering into the details, we only signalize that the answer depends essentially on $p_{0}$ and $p_{1}$ and has the simplest form in the case of powertype weights $w_{1}$ and $w_{2}$. Specifically (see Corollary 8), if $1 \leq p_{0} \leq p_{1}<\infty$ and either (a) $l>p_{0}-1$ and $m \leq 0$, or (b) $l>p_{0}-1, l>m$, and $0 \leq m<p_{1}-1$, then (0.4) is fulfilled with $w_{0}(x)=x^{l}$ and $w_{1}(x)=x^{m}$ if and only if $\theta \neq \frac{p_{1}\left(l-p_{0}+1\right)}{p_{1} l-p_{0} m+p_{1}-p_{0}}$.

For general weights, the situation is different. For instance, it is shown (see Corollary 7) that for all numbers $0 \leq a \leq b \leq c \leq d \leq 1$ there exist weights $w_{0}(x)$ and $w_{1}(x)$ such that, for $p_{0}=p_{1}=1$ and $q \in[1, \infty)$, relation $(0.4)$ is true if and only if $\theta \in$ $(0, a) \cup(b, c) \cup(d, 1)$. Thus, the values of $\theta$ for which the natural interpolation identity (0.1) fails may fill one or two arbitrary subsegments of $(0,1)$ (cf. [1, Corollary 2, Remark 8]).

Surely, a most natural approach to the subspace interpolation problem is via calculation of the $\mathcal{K}$-functional for the couple of subspaces in question; see [1, 8, 12. This problem proves to be interesting in itself and is treated in $\S 3$. In Theorem 3 an expression for $\mathcal{K}\left(t, x ; X_{0} \cap N, X_{1} \cap N\right)$ is found under the condition that the functional $\psi$ in question is defined on the set $\left\{y_{0}(t) \in X_{0}: y \in X_{0} \cap N+X_{1} \cap N, t>0\right\}$, where $y_{0}(t)$ is the first summand in an optimal decomposition $y=y_{0}(t)+y_{1}(t), y_{i}(t) \in X_{i}(i=0,1)$, i.e., in a decomposition for which

$$
\frac{1}{2}\left(\left\|y_{0}(t)\right\|_{X_{0}}+t\left\|y_{1}(t)\right\|_{X_{1}}\right) \leq \mathcal{K}\left(t, y ; X_{0}, X_{1}\right) \leq\left\|y_{0}(t)\right\|_{X_{0}}+t\left\|y_{1}(t)\right\|_{X_{1}} \quad(t>0) .
$$

Note that this condition is fulfilled in the case of the weighted $L_{p}$-spaces that were treated in [1]. Therefore, the theorem proved in the present paper extends the expressions for $\mathcal{K}\left(t, f ; L_{p}\left(w_{0}\right) \cap N, L_{p}\left(w_{1}\right) \cap N\right)$ obtained in [1] by direct inspection. In conclusion, we mention that a similar result for the couple $\left(Y_{0}, X_{1}\right), Y_{0}=\operatorname{Ker} \psi, \psi \in X_{0}^{*}$, was obtained by Wallsten [8] (see also [12, Theorem 2.1]).

Definitions and notation. Let $\left(X_{0}, X_{1}\right)$ be an arbitrary normed couple. The definitions of the Peetre $\mathcal{K}$-functional $\mathcal{K}\left(t, x ; X_{0}, X_{1}\right)\left(x \in X_{0}+X_{1}, t>0\right)$ and of the real interpolation spaces $X_{\theta, q}=\left(X_{0}, X_{1}\right)_{\theta, q}(0<\theta<1,1 \leq q<\infty)$ were given at the beginning of the paper. Analyzing the proof of the equivalence theorem [2, §3], we see that it remains true for normed (rather than merely for Banach) couples. Thus, the space $X_{\theta, q}$ (with an equivalent norm) can be defined in terms of the $\mathcal{J}$-functional

$$
\mathcal{J}\left(t, x ; X_{0}, X_{1}\right)=\max \left\{\|x\|_{X_{0}}, t\|x\|_{X_{1}}\right\} \quad\left(x \in X_{0} \cap X_{1}\right) .
$$

Specifically, $X_{\theta, q}$ consists of all $x \in X_{0}+X_{1}$ representable in the form

$$
\left.x=\sum_{k \in \mathbb{Z}} 2^{\theta k} x_{k} \text { (convergence in } X_{0}+X_{1}\right)
$$

with the norm

$$
\inf \left\{\sum_{k \in \mathbb{Z}}\left(\mathcal{J}\left(2^{k}, x_{k} ; X_{0}, X_{1}\right)\right)^{q}\right\}^{1 / q},
$$

where the infimum is taken over all representations indicated above.

Next, it will be tacitly assumed that $X_{0} \cap X_{1}$ is dense in $X_{0}$ and in $X_{1}$. Therefore, we may consider the Banach couple $\left(X_{0}^{*}, X_{1}^{*}\right)$ of conjugate spaces, and if $\psi \in\left(X_{0} \cap X_{1}\right)^{*}$, then $\psi \in X_{0}^{*}+X_{1}^{*}$ (see $\left.[2, \S 3.7]\right)$. The function $k(t)=\mathcal{K}\left(t, \psi ; X_{0}^{*}, X_{1}^{*}\right)$ will play a crucial role in what follows; we have

$$
k(t)=\sup \left\{|\psi(x)|: x \in X_{0} \cap X_{1}, \mathcal{J}\left(t^{-1}, x ; X_{0}, X_{1}\right) \leq 1\right\}
$$

(see [2, §3.7]). 
We introduce the functions

$$
M(t)=\sup _{s>0} \frac{k(t s)}{k(s)}, \quad M_{0}(t)=\sup _{0<s \leq \min (1,1 / t)} \frac{k(t s)}{k(s)}, \quad M_{\infty}(t)=\sup _{s \geq \max (1,1 / t)} \frac{k(t s)}{k(s)},
$$

which are semimultiplicative for $t>0$, and so the following numbers are well defined:

$$
\begin{aligned}
\alpha & =\lim _{t \rightarrow 0} \frac{\log _{2} M(t)}{\log _{2} t}, \quad \beta=\lim _{t \rightarrow \infty} \frac{\log _{2} M(t)}{\log _{2} t}, \\
\alpha_{0} & =\lim _{t \rightarrow 0} \frac{\log _{2} M_{0}(t)}{\log _{2} t}, \quad \beta_{\infty}=\lim _{t \rightarrow \infty} \frac{\log _{2} M_{\infty}(t)}{\log _{2} t} .
\end{aligned}
$$

These numbers are called the dilation indices of $k(t)$. It is easily seen that

$$
0 \leq \alpha \leq \min \left(\alpha_{0}, \beta_{\infty}\right) \leq \max \left(\alpha_{0}, \beta_{\infty}\right) \leq \beta \leq 1 .
$$

Now, putting $\mu_{n}=\left(k\left(2^{-n}\right)\right)^{-1}(n \in \mathbb{Z})$, we see that $\mu_{n}>0$, and, since $k(t)$ is monotone increasing and concave on $(0, \infty)$, also

$$
\mu_{n} \leq \mu_{n+1} \leq 2 \mu_{n} \quad(n \in \mathbb{Z}) .
$$

Furthermore,

$$
\begin{aligned}
\alpha & =-\lim _{n \rightarrow \infty} \frac{1}{n} \log _{2} \sup _{k \in \mathbb{Z}} \frac{\mu_{k}}{\mu_{n+k}}, & \beta & =\lim _{n \rightarrow \infty} \frac{1}{n} \log _{2} \sup _{k \in \mathbb{Z}} \frac{\mu_{k}}{\mu_{k-n}}, \\
\alpha_{0} & =-\lim _{n \rightarrow \infty} \frac{1}{n} \log _{2} \sup _{k \geq 0} \frac{\mu_{k}}{\mu_{n+k}}, & \beta_{\infty} & =\lim _{n \rightarrow \infty} \frac{1}{n} \log _{2} \sup _{k \leq 0} \frac{\mu_{k}}{\mu_{k-n}} .
\end{aligned}
$$

Let $e_{k}(k \in \mathbb{Z})$ denote the standard unit vectors in a sequence space, and let $l_{q}(\mu)$ be the space of two-sided numerical sequences $a=\left(a_{k}\right)_{k=-\infty}^{\infty}$ with the norm

$$
\|a\|_{q, \mu}=\left\{\sum_{k \in \mathbb{Z}}\left|a_{k}\right|^{q} \mu_{k}^{q}\right\}^{1 / q} .
$$

In what follows, we shall study the shift operator $S\left(a_{k}\right)=\left(a_{k-1}\right)$, its inverse $S^{-1}$, and also the operators $T_{\theta}=S-2^{\theta} I(0<\theta<1)$, where $I$ is the identity mapping. By $(0.7)$, both $S$ and $S^{-1}$ are bounded on $l_{q}(\mu)$, and $\|S\| \leq 2,\left\|S^{-1}\right\| \leq 1$. Next, by $P_{+}$and $P_{-}$ we denote the projections

$$
P_{+}\left(a_{k}\right)=\sum_{k=1}^{\infty} a_{k} e_{k}, \quad P_{-}\left(a_{k}\right)=\sum_{k=-1}^{-\infty} a_{k} e_{k},
$$

and $r(T)$ stands for the spectral radius of an operator $T$ on $l_{q}(\mu)$.

Finally, writing $F_{1} \asymp F_{2}$ we mean that there exist two constants $c>0, C>0$ with $c F_{1} \leq F_{2} \leq C F_{1}$. Usually, $c$ and $C$ do not depend on all (or some) of the arguments of the functions $F_{1}$ and $F_{2}$.

\section{§1. Auxiliary Results}

Lemma 1. We have $r(S)=2^{\beta}, r\left(S^{-1}\right)=2^{-\alpha}, r\left(S P_{-}\right)=2^{\beta_{\infty}}$, and $r\left(S^{-1} P_{+}\right)=2^{-\alpha_{0}}$.

Proof. Since $S^{n}\left(a_{k}\right)=\left(a_{k-n}\right)$ and $S^{-n}\left(a_{k}\right)=\left(a_{k+n}\right)$, we have

$$
\left\|S^{n}\right\|_{l_{q}(\mu) \rightarrow l_{q}(\mu)}=\sup _{k \in \mathbb{Z}} \frac{\mu_{k}}{\mu_{k-n}}, \quad\left\|S^{-n}\right\|_{l_{q}(\mu) \rightarrow l_{q}(\mu)}=\sup _{k \in \mathbb{Z}} \frac{\mu_{k}}{\mu_{k+n}} .
$$


Thus, by (0.8), we see that

$$
\begin{gathered}
r(S)=\lim _{n \rightarrow \infty}\left(\sup _{k \in \mathbb{Z}} \frac{\mu_{k}}{\mu_{k-n}}\right)^{1 / n}=2^{\beta}, \\
r\left(S^{-1}\right)=\lim _{n \rightarrow \infty}\left(\sup _{k \in \mathbb{Z}} \frac{\mu_{k}}{\mu_{k+n}}\right)^{1 / n}=2^{-\alpha} .
\end{gathered}
$$

Similarly,

$$
\left(S P_{-}\right)^{n}\left(a_{k}\right)=\sum_{k=-n}^{-\infty} a_{k} e_{k+n}, \quad\left(S^{-1} P_{+}\right)^{n}\left(a_{k}\right)=\sum_{k=n}^{\infty} a_{k} e_{k-n}, \quad n=1,2, \ldots,
$$

and, therefore,

$$
\left\|\left(S P_{-}\right)^{n}\right\|_{l_{q}(\mu) \rightarrow l_{q}(\mu)}=\sup _{k \leq 0} \frac{\mu_{k}}{\mu_{k-n}}, \quad\left\|\left(S^{-1} P_{+}\right)^{n}\right\|_{l_{q}(\mu) \rightarrow l_{q}(\mu)}=\sup _{k \geq 0} \frac{\mu_{k}}{\mu_{k+n}} .
$$

This results in

$$
\begin{gathered}
r\left(S P_{-}\right)=\lim _{n \rightarrow \infty}\left(\sup _{k \leq 0} \frac{\mu_{k}}{\mu_{k-n}}\right)^{1 / n}=2^{\beta_{\infty}}, \\
r\left(S^{-1} P_{+}\right)=\lim _{n \rightarrow \infty}\left(\sup _{k \geq 0} \frac{\mu_{k}}{\mu_{k+n}}\right)^{1 / n}=2^{-\alpha_{0}} .
\end{gathered}
$$

The next key statement about properties of $T_{\theta}=S-2^{\theta} I$ ( $I$ is the identity) is similar to Lemma 2.2 in [3]. However, there is an important point of difference. In [3] the functional $\psi$ belonged to $X^{*}$, which resulted in $\inf _{n \in \mathbb{Z}} \mu_{n}>0$. The latter condition implies that $T_{\theta}$ is injective for every $\theta \in(0,1)$. In the present more general setting, where $\psi \in\left(X_{0} \cap X_{1}\right)^{*}$, the operator $T_{\theta}$ may fail to be injective because $\inf _{n \in \mathbb{Z}} \mu_{n}$ may happen to be zero.

Lemma 2. Suppose $0<\theta<1$ and $1 \leq q<\infty$. Then $T_{\theta}$ is an isomorphic embedding of $l_{q}(\mu)$ into $l_{q}(\mu)$ if and only if $\theta \in(0, \alpha) \cup\left(\beta_{\infty}, \alpha_{0}\right) \cup(\beta, 1)$.

Moreover, if $\theta \in(0, \alpha) \cup(\beta, 1)$, then $\operatorname{Im} T_{\theta}=l_{q}(\mu)$; if $\theta \in\left(\beta_{\infty}, \alpha_{0}\right)$, then $\operatorname{Im} T_{\theta}$ is the closed subspace of codimension 1 in $l_{q}(\mu)$ consisting of all $\left(a_{k}\right)_{k \in \mathbb{Z}} \in l_{q}(\mu)$ with

$$
\sum_{k \in \mathbb{Z}} 2^{k \theta} a_{k}=0
$$

Proof. First, if $\theta \in(\beta, 1)$, then Lemma 1 implies the inequality $2^{\theta}>r(S)$, whence $T_{\theta}$ is an automorphism of $l_{q}(\mu)$ onto $l_{q}(\mu)$. The same is true for $\theta \in(0, \alpha)$ because then $r\left(S^{-1}\right)<2^{-\theta}$ (again by Lemma 1 ) and

$$
T_{\theta}=2^{\theta} S\left(2^{-\theta} I-S^{-1}\right) \text {. }
$$

We show that neither $T_{\beta}$ nor $T_{\alpha}$ is an isomorphism of $l_{q}(\mu)$ onto $l_{q}(\mu)$. Indeed, for every $\lambda \in \mathbb{C}$ with $|\lambda|=1$ the operators $Q_{\lambda}\left(a_{i}\right)=\left(\lambda^{i} a_{i}\right)$ and $Q_{\lambda}^{\prime}\left(a_{i}\right)=\left(\lambda^{i+1} a_{i}\right)$ are isometries of $l_{q}(\mu)$. Since $S-\lambda 2^{\beta} I=Q_{\lambda}^{-1} T_{\beta} Q_{\lambda}^{\prime}$, the supposition that $T_{\beta}$ is an isomorphism onto $l_{q}(\mu)$ would imply that all operators $S-\lambda 2^{\beta} I(\lambda \in \mathbb{C},|\lambda|=1)$ have the same property. But this contradicts the relation $r(S)=2^{\beta}$. Similarly, the supposition that $T_{\alpha}$ is an isomorphism onto $l_{q}(\mu)$ would imply the same property for $S^{-1}-\lambda 2^{-\alpha} I$ (apply (1.2) with $\theta=\alpha$ and take the relation $S^{-1}-\lambda 2^{-\alpha} I=Q_{\lambda}^{\prime}\left(S^{-1}-2^{-\alpha} I\right) Q_{\lambda}^{-1}$ into account). This contradicts the fact that $r\left(S^{-1}\right)=2^{-\alpha}$.

Now we determine the possible form of $\operatorname{Im} T_{\theta}$. Since

$$
T_{\theta}\left(\sum_{i=0}^{n-1} 2^{(n-1-i) \theta} e_{i}\right)=e_{n}-2^{\theta n} e_{0},
$$


we have $e_{n}-2^{\theta n} e_{0} \in \operatorname{Im} T_{\theta}$ for all $n \in \mathbb{Z}$. Suppose $f_{\theta}$ is a linear functional vanishing at every $b \in \operatorname{Im} T_{\theta}$. Then $f_{\theta}\left(e_{n}\right)=2^{\theta n} C$. Thus, we may assume that $f_{\theta}$ corresponds to the sequence $\left(2^{\theta n}\right)_{n \in \mathbb{Z}}$.

Clearly, the condition $f_{\theta} \in l_{q}(\mu)^{*}$ is equivalent to the condition

$$
\sum_{k \in \mathbb{Z}} 2^{k q^{\prime} \theta} \mu_{k}^{-q^{\prime}}<\infty, \quad \text { where } \frac{1}{q^{\prime}}+\frac{1}{q}=1
$$

(with the usual modification if $q^{\prime}=\infty$ ). If $f_{\theta}$ is a bounded functional, the Hahn-Banach theorem and the preceding arguments imply that

$$
\overline{\operatorname{Im} T_{\theta}}=\operatorname{Ker} f_{\theta} .
$$

On the other hand, if $f_{\theta} \notin l_{q}(\mu)^{*}$, then

$$
\overline{\operatorname{Im} T_{\theta}}=l_{q}(\mu) .
$$

We show that if $\theta \in\left(\beta_{\infty}, \alpha_{0}\right)$, then the functional $f_{\theta}$ is bounded and $\operatorname{Im} T_{\theta}$ is closed. Let $\beta_{\infty}+\varepsilon<\theta<\alpha_{0}-\varepsilon$ for some $\varepsilon>0$. By (0.8), we have

$$
\sup _{k \geq 0} \frac{\mu_{k}}{\mu_{k+n}} \leq C_{1} 2^{-n\left(\alpha_{0}-\varepsilon\right)}, \quad \sup _{k \leq 0} \frac{\mu_{k}}{\mu_{k-n}} \leq C_{2} 2^{n\left(\beta_{\infty}+\varepsilon\right)} \quad(n=1,2, \ldots),
$$

whence

$$
\mu_{n}^{-1} \leq \mu_{0}^{-1} C_{1} 2^{-n\left(\alpha_{0}-\varepsilon\right)}, \quad \mu_{-n}^{-1} \leq \mu_{0}^{-1} C_{2} 2^{n\left(\beta_{\infty}+\varepsilon\right)} \quad(n=1,2, \ldots) .
$$

Therefore,

$$
\sum_{n \in \mathbb{Z}} 2^{n \theta} \mu_{n}^{-1} \leq \mu_{0}^{-1} C_{1} \sum_{n=0}^{\infty} 2^{n\left(\theta-\alpha_{0}+\varepsilon\right)}+\mu_{0}^{-1} C_{2} \sum_{n=1}^{\infty} 2^{-n\left(\theta-\beta_{\infty}-\varepsilon\right)}<\infty,
$$

i.e., (1.3) is true for every $1 \leq q<\infty$, yielding $f_{\theta} \in l_{q}(\mu)^{*}$. In order to prove that $\operatorname{Im} T_{\theta}$ is closed, we represent $l_{q}(\mu)$ as follows:

$$
l_{q}(\mu)=E_{-}+E_{0}+E_{+},
$$

where $E_{-}=\left[\left\{e_{n}\right\}_{n \leq-1}\right]_{q, \mu}, E_{0}=\left[\left\{e_{0}\right\}\right]_{q, \mu}$, and $E_{+}=\left[\left\{e_{n}\right\}_{n \geq 1}\right]_{q, \mu}$ (here $[F]_{q, \mu}$ is the closed linear span of a set $F$ in the space $\left.l_{q}(\mu)\right)$. Since

$$
\operatorname{Im} T_{\theta}=T_{\theta}\left(E_{-}\right)+T_{\theta}\left(E_{0}\right)+T_{\theta}\left(E_{+}\right)
$$

$T_{\theta}\left(E_{-}\right) \cap T_{\theta}\left(E_{+}\right)=\{0\}$, and the space $T_{\theta}\left(E_{0}\right)$ is one-dimensional, we see that it suffices to prove that $T_{\theta}\left(E_{-}\right)$and $T_{\theta}\left(E_{+}\right)$are closed.

Since $\beta_{\infty}<\theta<\alpha_{0}$, and $r\left(S P_{-}\right)=2^{\beta_{\infty}}, r\left(S^{-1} P_{+}\right)=2^{-\alpha_{0}}$ by Lemma 1 , it follows that the operators $S P_{-}-2^{\theta} I$ and $S^{-1} P_{+}-2^{-\theta} I$ map $l_{q}(\mu)$ onto $l_{q}(\mu)$ isomorphically. Therefore, the image $T_{\theta}\left(E_{-}\right)=\left(S P_{-}-2^{\theta} I\right)\left(E_{-}\right)$of the closed set $E_{-}$is also closed. Similarly, $T_{\theta}\left(E_{+}\right)$is closed because $S$ is an isomorphism of $l_{q}(\mu)$ and $T_{\theta}\left(E_{+}\right)=-2^{\theta} S\left(S^{-1} P_{+}-2^{-\theta} I\right)\left(E_{+}\right)$.

Furthermore, it is easily seen that $T_{\theta}$ is injective for $\theta \in\left(0, \alpha_{0}\right) \cup\left(\beta_{\infty}, 1\right)$. Indeed, the relation $T_{\theta} a=0$ means that $a_{n-1}=2^{\theta} a_{n}$, i.e., $a_{n}=2^{-n \theta} a_{0}(n \in \mathbb{Z})$. Since $a \in l_{q}(\mu)$, we must have

$$
\left|a_{0}\right|^{q} \sum_{k \in \mathbb{Z}} 2^{-k q \theta} \mu_{k}^{q}<\infty .
$$

If, for instance, $\theta<\alpha_{0}$, then the first inequality in (1.6) with $0<\varepsilon<\alpha_{0}-\theta$ shows that (1.7) is true only if $a_{0}=0$, i.e., $a=0$. But if $\theta>\beta_{\infty}$, we can argue similarly by using the second inequality in (1.6) with $0<\varepsilon<\theta-\beta_{\infty}$.

Thus, for $\beta_{\infty}<\theta<\alpha_{0}$ the operator $T_{\theta}$ maps $l_{q}(\mu)$ isomorphically onto the subspace of codimension 1 consisting of all $\left(a_{k}\right) \in l_{q}(\mu)$ that satisfy (1.1). 
Next, suppose $\theta \notin(0, \alpha] \cup[\beta, 1)$ and $T_{\theta}: l_{q}(\mu) \rightarrow \operatorname{Im} T_{\theta}$ is an isomorphism. Then there exists $c>0$ such that

$$
\left\|T_{\theta} a\right\|_{l_{q}(\mu)} \geq c\|a\|_{l_{q}(\mu)}
$$

for all $a \in l_{q}(\mu)$.

For arbitrary (for the moment) $n \in \mathbb{N}$ and $k \in \mathbb{Z}$, put $a=\left(I+2^{-\theta} S+\cdots+2^{-n \theta} S^{n}\right)^{2} e_{k}$. Direct inspection shows that $a \geq n 2^{-n \theta} e_{k+n}$, whence

$$
\|a\|_{l_{q}(\mu)} \geq n 2^{-n \theta} \mu_{k+n} .
$$

We estimate the quantity $\left\|T_{\theta}^{2} a\right\|_{l_{q}(\mu)}$ from above. First,

$$
\begin{aligned}
T_{\theta}^{2}(I & \left.+2^{-\theta} S+\cdots+2^{-n \theta} S^{n}\right)^{2} \\
& =2^{\theta}\left(S-2^{\theta} I\right)\left(2^{-(n+1) \theta} S^{n+1}-I\right)\left(I+2^{-\theta} S+\cdots+2^{-n \theta} S^{n}\right) \\
& =2^{2 \theta} I-2^{1-(n-1) \theta} S^{n+1}+2^{-2 n \theta} S^{2 n+2} .
\end{aligned}
$$

Consequently,

$$
T_{\theta}^{2} a=2^{2 \theta} e_{k}-2^{1-(n-1) \theta} e_{n+k+1}+2^{-2 n \theta} e_{2 n+k+2},
$$

and inequalities (0.7) imply that

$$
\begin{aligned}
\left\|T_{\theta}^{2} a\right\|_{l_{q}(\mu)} & =\left(2^{2 \theta} \mu_{k}^{q}-2^{q} 2^{-(n-1) q \theta} \mu_{n+k+1}^{q}+2^{-2 q n \theta} \mu_{2 n+k+2}^{q}\right)^{1 / q} \\
& \leq 4 \mu_{k}+8 \cdot 2^{-n \theta} \mu_{n+k}+4 \cdot 2^{-2 n \theta} \mu_{2 n+k} .
\end{aligned}
$$

Therefore,

$$
\left\|T_{\theta}^{2} a\right\|_{l_{q}(\mu)}-8 \cdot 2^{-n \theta} \mu_{n+k} \leq 4\left(\mu_{k}+2^{-2 n \theta} \mu_{2 n+k}\right) \leq 8 \max \left(\mu_{k}, 2^{-2 n \theta} \mu_{2 n+k}\right) .
$$

Now we choose $n$ with $n c^{2}>16$. Then (1.8) and (1.9) imply that

$$
\left\|T_{\theta}^{2} a\right\|_{l_{q}(\mu)} \geq c^{2} n 2^{-n \theta} \mu_{n+k}
$$

whence

$$
2^{-n \theta} \mu_{n+k}<\max \left(\mu_{k}, 2^{-2 n \theta} \mu_{2 n+k}\right)
$$

or, equivalently,

$$
\nu_{n+k}<\max \left(\nu_{k}, \nu_{2 n+k}\right) \quad(k \in \mathbb{Z})
$$

with $\nu_{n}=2^{-n \theta} \mu_{n}$.

Since $\theta<\beta$ by assumption, for the number $n$ chosen above we can find $k \in \mathbb{Z}$ with $\mu_{n+k}>2^{n \theta} \mu_{k}$, i.e., $\nu_{k+n}>\nu_{k}$. Therefore, $\nu_{k+2 n}>\nu_{k+n}$ by (1.10). Substituting $k+n$ for $k$ in (1.10) and arguing in the same way, we obtain $\nu_{k+3 n}>\nu_{k+2 n}$, and so on. Thus, the sequence $\left(\nu_{k+r n}\right)_{r=0}^{\infty}$ is monotone increasing.

Let $j \geq k$ and $m \geq n$. We find $0 \leq r_{1} \leq r_{2}$ with

$$
k+\left(r_{1}-1\right) n \leq j \leq k+r_{1} n, \quad k+r_{2} n \leq j+m \leq k+\left(r_{2}+1\right) n .
$$

Then

$$
\frac{\mu_{j+m}}{\mu_{j}} \geq \frac{\mu_{k+r_{2} n}}{\mu_{k+r_{1} n}}=\frac{2^{r_{2} n \theta} \nu_{k+r_{2} n}}{2^{r_{1} n \theta} \nu_{k+r_{1} n}} \geq 2^{n\left(r_{2}-r_{1}\right) \theta} .
$$

Since $m-2 n \leq\left(r_{2}-r_{1}\right) n$, we see that

$$
\frac{\mu_{j+m}}{\mu_{j}} \geq 2^{(m-2 n) \theta} \quad(j \geq k, m \geq n),
$$

that is,

$$
\sup _{j \geq k} \frac{\mu_{j}}{\mu_{j+m}} \leq C 2^{-m \theta} \quad(m \geq n) .
$$


But by (0.7), for $k>0$ and $0 \leq j \leq k$ we have

implying

$$
\frac{\mu_{j}}{\mu_{j+m}} \leq \frac{\mu_{k}}{\mu_{k+m}} \frac{\mu_{k+m}}{\mu_{j+m}} \leq 2^{k} \frac{\mu_{k}}{\mu_{k+m}},
$$

$$
\sup _{j \geq 0} \frac{\mu_{j}}{\mu_{j+m}} \leq C^{\prime} 2^{-m \theta} \quad(m \geq n) .
$$

Taking (0.8) into account, we obtain

$$
\theta \leq \alpha_{0}
$$

The inequality

$$
\theta \geq \beta_{\infty}
$$

is proved similarly. Indeed, for the element $b=\left(I+2^{-\theta} S+\cdots+2^{-n \theta} S^{n}\right)^{2} e_{k-2 n}$ we have

$$
\|b\|_{l_{q}(\mu)} \geq n 2^{-n \theta} \mu_{k-n} .
$$

Since

$$
T_{\theta}^{2} b=2^{2 \theta} e_{k-2 n}-2^{1-(n-1) \theta} e_{k-n+1}+2^{-2 n \theta} e_{k+2},
$$

by (0.7) we have

$$
\begin{aligned}
\left\|T_{\theta}^{2} b\right\|_{l_{q}(\mu)} & \leq 2^{2 \theta} \mu_{k-2 n}+4 \cdot 2^{-n \theta} \mu_{k-n+1}+2^{-2 n \theta} \mu_{k+2} \\
& \leq 4 \mu_{k-2 n}+8 \cdot 2^{-n \theta} \mu_{k-n}+4 \cdot 2^{-2 n \theta} \mu_{k} .
\end{aligned}
$$

Again, if $n c^{2}>16$, then (1.8) and (1.13) imply that

$$
2^{-n \theta} \mu_{k-n}<\max \left(\mu_{k-2 n}, 2^{-2 n \theta} \mu_{k}\right),
$$

so that

$$
\nu_{k-n}<\max \left(\nu_{k}, \nu_{k-2 n}\right) \quad(k \in \mathbb{Z}),
$$

where $\nu_{n}=2^{-n \theta} \mu_{n}$, as before. If $\theta>\alpha$, then for $n$ chosen above there exists $k \in \mathbb{Z}$ with $\mu_{k-n}>2^{-n \theta} \mu_{k}$. Consequently, $\nu_{k}<\nu_{k-n}$. Applying (1.14), we obtain $\nu_{k-n}<\nu_{k-2 n}$. Repeating the arguments, we show that the sequence $\left(\nu_{k-r n}\right)_{r=0}^{\infty}$ is monotone increasing.

If $j \geq-k$ and $m \geq n$, there exist $0 \leq r_{1} \leq r_{2}$ with

$$
k-\left(r_{1}+1\right) n \leq-j \leq k-r_{1} n, \quad k-r_{2} n \leq-j-m \leq k-\left(r_{2}-1\right) n .
$$

Then

$$
\frac{\mu_{-j}}{\mu_{-m-j}} \leq \frac{\mu_{k-r_{1} n}}{\mu_{k-r_{2} n}}=\frac{2^{-r_{1} n \theta} \nu_{k-r_{1} n}}{2^{-r_{2} n \theta} \nu_{k-r_{2} n}} \leq 2^{n\left(r_{2}-r_{1}\right) \theta},
$$

and since $\left(r_{2}-r_{1}\right) n \leq m+2 n$, we obtain

$$
\sup _{j \geq-k} \frac{\mu_{-j}}{\mu_{-j-m}} \leq C 2^{m \theta} \quad(m \geq n) .
$$

As before, from this we deduce the estimate

$$
\sup _{j \geq 0} \frac{\mu_{-j}}{\mu_{-j-m}} \leq C^{\prime} 2^{m \theta} \quad(m \geq n) .
$$

This implies (1.12) by (0.8).

Summarizing, we see that the operator $T_{\theta}: l_{q}(\mu) \rightarrow \operatorname{Im} T_{\theta}$ is an isomorphism for $\theta \in(0, \alpha) \cup\left(\beta_{\infty}, \alpha_{0}\right) \cup(\beta, 1)$, and it is not an isomorphism for $\theta \in\left(\alpha, \beta_{\infty}\right) \cup\left(\alpha_{0}, \beta\right)$ by $(1.11)$ and (1.12). Furthermore, the operator $T_{\theta}$ is Fredholm with index 0 for $\theta \in(0, \alpha) \cup(\beta, 1)$ and it is Fredholm with index -1 for $\theta \in\left(\beta_{\infty}, \alpha_{0}\right)$. Since the set of all Fredholm operators with fixed index is open, and for $\theta=\alpha$ and $\theta=\beta$ the operator $T_{\theta}$ is not an isomorphism onto $l_{q}(\mu)$, the lemma is proved by (1.4) and (1.5). 
Remark 1. Lemma 2 and its proof show that, under the condition $\beta_{\infty} \leq \alpha_{0}$, the operator $T_{\theta}$ is an isomorphic embedding of $l_{q}(\mu)$ into $l_{q}(\mu)$ if and only if the image of $T_{\theta}$ is closed. Indeed, since $T_{\theta}$ is injective for $\theta \in\left(0, \alpha_{0}\right) \cup\left(\beta_{\infty}, 1\right)$, it suffices to consider the case where $\beta_{\infty}=\alpha_{0}$ and show that $T_{\alpha_{0}}$ must be injective if its image is closed. But otherwise $T_{\theta}$ is a Fredholm operator of constant index in some small neighborhood of $\alpha_{0}$; since it is injective for $\theta \neq \alpha_{0}$, it is an isomorphic embedding of $l_{q}(\mu)$ into $l_{q}(\mu)$. However, its index can only be equal to zero, i.e., $T_{\theta}\left(l_{q}(\mu)\right)=l_{q}(\mu)$. In particular, the index of $T_{\alpha_{0}}$ is also equal to zero, whence $T_{\alpha_{0}}\left(l_{q}(\mu)\right)$ is a closed subspace of codimension 1 in $l_{q}(\mu)$. But this implies (1.3); consequently, (1.7) may only be fulfilled if $a=0$, that is, $T_{\alpha_{0}}$ is injective, a contradiction.

The following simple statement is contained in fact in [15, Lemma 3.2]. We include it for completeness.

Lemma 3. For every Banach couple $\left(Y_{0}, Y_{1}\right)$, every $y \in Y_{0}+Y_{1}$, and $t \in(0,1]$ we have

$$
\frac{1}{4} \mathcal{K}\left(t, y ; Y_{0}, Y_{1}\right) \leq \mathcal{K}\left(t, y ; Y_{0}+Y_{1}, Y_{1}\right) \leq \mathcal{K}\left(t, y ; Y_{0}, Y_{1}\right)
$$

Proof. It suffices to verify the left-hand inequality. By the definition of the $\mathcal{K}$-functional, for every $t>0$ there is a representation $y=u^{t}+v^{t}$ with $u^{t} \in Y_{0}+Y_{1}, v^{t} \in Y_{1}$, and

$$
\mathcal{K}\left(t, y ; Y_{0}+Y_{1}, Y_{1}\right) \geq \frac{1}{2}\left(\left\|u^{t}\right\|_{Y_{0}+Y_{1}}+t\left\|v^{t}\right\|_{Y_{1}}\right) .
$$

For a similar reason, $u^{t}=u_{0}^{t}+u_{1}^{t}$, where $u_{0}^{t} \in Y_{0}, u_{1}^{t} \in Y_{1}$ and

$$
\left\|u^{t}\right\|_{Y_{0}+Y_{1}} \geq \frac{1}{2}\left(\left\|u_{0}^{t}\right\|_{Y_{0}}+\left\|u_{1}^{t}\right\|_{Y_{1}}\right)
$$

Since $t \leq 1$, it follows that

$$
\mathcal{K}\left(t, y ; Y_{0}+Y_{1}, Y_{1}\right) \geq \frac{1}{4}\left(\left\|u_{0}^{t}\right\|_{Y_{0}}+t\left\|u_{1}^{t}+v^{t}\right\|_{Y_{1}}\right) \geq \frac{1}{4} \mathcal{K}\left(t, y ; Y_{0}, Y_{1}\right) .
$$

Lemma 4. For every Banach couple $\left(X_{0}, X_{1}\right)$ and arbitrary $0<\theta<1$ and $1 \leq q \leq \infty$ we have

$$
\left(X_{0} \cap X_{1}, X_{1}\right)_{\theta, q}=\left(X_{0}, X_{1}\right)_{\theta, q} \cap X_{1}
$$

and

$$
\left(X_{0}, X_{0} \cap X_{1}\right)_{\theta, q}=\left(X_{0}, X_{1}\right)_{\theta, q} \cap X_{0} .
$$

Proof. Since $\left(A_{0}, A_{1}\right)_{\theta, q}=\left(A_{1}, A_{0}\right)_{1-\theta, q}$, it suffices to verify (1.15). The embedding

$$
\left(X_{0} \cap X_{1}, X_{1}\right)_{\theta, q} \subset\left(X_{0}, X_{1}\right)_{\theta, q} \cap X_{1}
$$

is obvious. Thus, the lemma will be proved if we show that

$$
\mathcal{K}\left(t, x ; X_{0} \cap X_{1}, X_{1}\right) \leq 2\left(\mathcal{K}\left(t, x ; X_{0}, X_{1}\right)+\min (1, t)\|x\|_{X_{1}}\right)
$$

for all $x \in X_{1}$ and $t>0$.

If $0<t \leq 1$, then $\mathcal{K}\left(t, x ; X_{0} \cap X_{1}, X_{1}\right)=t\|x\|_{X_{1}}$, and (1.17) is fulfilled. Now, let $t>1$. For $x \in X_{1}$ and $\varepsilon>0$, we find a decomposition $x=x_{0}+x_{1}, x_{i} \in X_{i}(i=0,1)$, such that

$$
\mathcal{K}\left(2 t, x ; X_{0}, X_{1}\right) \geq(1-\varepsilon)\left(\left\|x_{0}\right\|_{X_{0}}+2 t\left\|x_{1}\right\|_{X_{1}}\right) .
$$


Then $x_{0} \in X_{0} \cap X_{1}$. Since the $\mathcal{K}$-functional is concave in $t$, the preceding inequality implies that

$$
\begin{aligned}
\mathcal{K}\left(t, x ; X_{0} \cap X_{1}, X_{1}\right) & \leq \max \left(\left\|x_{0}\right\|_{X_{0}},\left\|x_{0}\right\|_{X_{1}}\right)+t\left\|x_{1}\right\|_{X_{1}} \\
& \leq\left\|x_{0}\right\|_{X_{0}}+(t+1)\left\|x_{1}\right\|_{X_{1}}+\|x\|_{X_{1}} \\
& \leq(1-\varepsilon)^{-1} \mathcal{K}\left(2 t, x ; X_{0}, X_{1}\right)+\|x\|_{X_{1}} \\
& \leq \frac{2}{1-\varepsilon}\left(\mathcal{K}\left(t, x ; X_{0}, X_{1}\right)+\|x\|_{X_{1}}\right) .
\end{aligned}
$$

Since $\varepsilon>0$ is arbitrary, (1.17) follows.

A statement close to the above was obtained in [7].

\section{§2. Real interpolation FOR COUPLES OF INTERSECTIONS}

In what follows, $\left(X_{0}, X_{1}\right)$ is a Banach couple with $X_{0} \cap X_{1}$ dense both in $X_{0}$ and in $X_{1}$, and $X_{\theta, q}=\left(X_{0}, X_{1}\right)_{\theta, q}(0<\theta<1,1 \leq q<\infty)$. Let $\psi$ be a linear functional with $\psi \in\left(X_{0} \cap X_{1}\right)^{*}$, and let $\alpha, \beta, \alpha_{0}$, and $\beta_{\infty}$ be the dilation indices of the function $k(t)=\mathcal{K}\left(t, \psi, X_{0}^{*}, X_{1}^{*}\right)$.

We denote $N=\operatorname{Ker} \psi$ and $N_{\theta, q}=\left(N_{0}, N_{1}\right)_{\theta, q}$, where $N_{i}$ is the space $N$ endowed with the norm of $X_{i}(i=0,1)$. Clearly, $N_{\theta, q} \subset X_{\theta, q}$ and $\|x\|_{X_{\theta, q}} \leq\|x\|_{N_{\theta, q}}\left(x \in N_{\theta, q}\right)$.

Theorem 1. If

$$
\theta \in(0, \alpha) \cup\left(\beta_{\infty}, \alpha_{0}\right) \cup(\beta, 1),
$$

then the norms of the spaces $N_{\theta, q}$ and $X_{\theta, q}$ are equivalent on $N$. Under the condition $\beta_{\infty} \leq \alpha_{0}$, the converse is also true: if the norms of $N_{\theta, q}$ and $X_{\theta, q}$ are equivalent on $N$, then (2.1) is fulfilled.

Moreover, if $\theta \in(0, \alpha) \cup(\beta, 1)$, then $N_{\theta, q}$ is dense in $X_{\theta, q}$, and if $\theta \in\left(\beta_{\infty}, \alpha_{0}\right)$, then $N_{\theta, q}$ is dense in some subspace of codimension 1 in $X_{\theta, q}$.

Proof. First, suppose (2.1) is fulfilled. In the previous notation, by Lemma 2 we have

$$
\|a\|_{l_{q}(\mu)} \leq B\left\|T_{\theta} a\right\|_{l_{q}(\mu)}, \quad a \in l_{q}(\mu),
$$

with some $B>0$. Furthermore, if a) $\theta \in(0, \alpha) \cup(\beta, 1)$, then $\operatorname{Im} T_{\theta}=l_{q}(\mu)$, and if b) $\theta \in\left(\beta_{\infty}, \alpha_{0}\right)$, then $\operatorname{Im} T_{\theta}=\operatorname{Ker} f_{\theta}$, where the functional $f_{\theta} \in l_{q}(\mu)^{*}$ is defined by

$$
f_{\theta}(a)=\sum_{n \in \mathbb{Z}} 2^{n \theta} a_{n}, \quad a=\left(a_{n}\right) \in l_{q}(\mu) .
$$

In case b), condition (1.3) is fulfilled, i.e.,

$$
\sum_{k \in \mathbb{Z}} 2^{k q^{\prime} \theta} \mathcal{K}\left(2^{-k}, \psi\right)^{q^{\prime}}=C_{0}^{q^{\prime}}<\infty, \quad \frac{1}{q^{\prime}}+\frac{1}{q}=1
$$

(here and below $\left.\mathcal{K}(t, \psi):=\mathcal{K}\left(t, \psi, X_{0}^{*}, X_{1}^{*}\right), \mathcal{J}(t, x):=\mathcal{J}\left(t, x, X_{0}, X_{1}\right)\right)$; therefore, $\psi$ can be extended up to a functional $\tilde{\psi} \in X_{\theta, q}^{*}$ [16, Theorem 3.7.2].

Now, let $x \in X_{\theta, q}$ with $\|x\|_{X_{\theta, q}}=1$. Then there is a sequence $\left\{x_{k}\right\} \subset X_{0} \cap X_{1}$ such that

$$
x=\sum_{k \in \mathbb{Z}} 2^{k \theta} x_{k}, \quad\left\|\left(\mathcal{J}\left(2^{k}, x_{k}\right)\right)_{k}\right\|_{q} \leq 2 .
$$

Since $\psi \in\left(X_{0} \cap X_{1}\right)^{*}$ by assumption, formula (0.6) implies that

$$
\left|\psi\left(x_{k}\right)\right| \leq \mathcal{K}\left(2^{-k}, \psi\right) \mathcal{J}\left(2^{k}, x_{k}\right)=\mu_{k}^{-1} \mathcal{J}\left(2^{k}, x_{k}\right) \quad(k \in \mathbb{Z}) .
$$


By (2.3), it follows that

$$
\left(\sum_{k \in \mathbb{Z}}\left|\psi\left(x_{k}\right)\right|^{q} \mu_{k}^{q}\right)^{1 / q} \leq\left\|\left(\mathcal{J}\left(2^{k}, x_{k}\right)\right)_{k}\right\|_{q} \leq 2,
$$

i.e., $\left(\psi\left(x_{k}\right)\right) \in l_{q}(\mu)$.

In case b) we assume additionally that $x \in \operatorname{Ker} \tilde{\psi}$. Since the series in (2.3) converges in $X_{\theta, q}$ and $\tilde{\psi} \in X_{\theta, q}{ }^{*}$, we have $\sum_{k \in \mathbb{Z}} 2^{\theta k} \psi\left(x_{k}\right)=0$. Now, Lemma 2 shows that $\left(\psi\left(x_{k}\right)\right) \in$ $\operatorname{Im} T_{\theta}$ both in case a) and in case b). Thus, there exists a sequence $a \in l_{q}(\mu)$ such that $T_{\theta} a=\left(\psi\left(x_{k}\right)\right)$ and, moreover, $\|a\|_{l_{q}(\mu)} \leq 2 B$ by $(2.2)$ and $(2.4)$.

Using (0.6) once again, we find a sequence $\left\{u_{n}^{\prime}\right\} \in X_{0} \cap X_{1}$ such that $\mathcal{J}\left(2^{n}, u_{n}^{\prime}\right) \leq 2$ and $\psi\left(u_{n}^{\prime}\right)=\mu_{n}^{-1}(n \in \mathbb{Z})$. Putting $u_{n}=a_{n} \mu_{n} u_{n}^{\prime}$, we see that $u_{n} \in X_{0} \cap X_{1}, \mathcal{J}\left(2^{n}, u_{n}\right) \leq$ $2\left|a_{n}\right| \mu_{n}$, and $\psi\left(u_{n}\right)=a_{n}(n \in \mathbb{Z})$. Consequently,

$$
\left\|\left(\mathcal{J}\left(2^{n}, u_{n}\right)\right)_{n}\right\|_{q} \leq 2\|a\|_{l_{q}(\mu)} \leq 4 B .
$$

In particular, it follows immediately that the series $\sum_{k \in \mathbb{Z}} 2^{\theta k} u_{k}$ converges in $X_{0}+X_{1}$. Indeed,

$$
\begin{aligned}
\left\|\sum_{n \geq N} 2^{\theta n} u_{n}\right\|_{X_{1}} & \leq \sum_{n \geq N} 2^{(\theta-1) n} 2^{n}\left\|u_{n}\right\|_{X_{1}} \\
& \leq\left(\sum_{n \geq N}\left(2^{n}\left\|u_{n}\right\|_{X_{1}}\right)^{q}\right)^{1 / q}\left(\sum_{n \geq N} 2^{n(\theta-1) q^{\prime}}\right)^{1 / q^{\prime}} \\
& \leq C\left(\sum_{n \geq N}\left(\mathcal{J}\left(2^{n}, u_{n}\right)\right)^{q}\right)^{1 / q} \rightarrow 0 \quad \text { as } N \rightarrow \infty
\end{aligned}
$$

and similarly

$$
\begin{aligned}
\left\|\sum_{n \leq-N} 2^{\theta n} u_{n}\right\|_{X_{0}} & \leq \sum_{n \leq-N} 2^{\theta n}\left\|u_{n}\right\|_{X_{0}} \\
& \leq C^{\prime}\left(\sum_{n \leq-N}\left(\mathcal{J}\left(2^{n}, u_{n}\right)\right)^{q}\right)^{1 / q} \rightarrow 0 \text { as } N \rightarrow \infty .
\end{aligned}
$$

Therefore, putting $v_{n}=u_{n-1}-2^{\theta} u_{n}$, we obtain $\sum_{k \in \mathbb{Z}} 2^{\theta k} v_{k}=0$, whence

$$
x=\sum_{k \in \mathbb{Z}} 2^{\theta k} y_{k}
$$

with $y_{k}=x_{k}-v_{k}$, by (2.3). Furthermore,

$$
\psi\left(y_{k}\right)=\psi\left(x_{k}\right)-\psi\left(u_{k-1}\right)+2^{\theta} \psi\left(u_{k}\right)=\left(T_{\theta} a\right)_{k}-a_{k-1}+2^{\theta} a_{k}=0,
$$

i.e., $y_{k} \in N(k \in \mathbb{Z})$. Since the series in (2.6) converges in $X_{\theta, q}$, the second part of the theorem is proved.

Next, by (2.3) and (2.5) we obtain

$$
\begin{aligned}
\left\|\left(\mathcal{J}\left(2^{n}, y_{n}\right)\right)_{n}\right\|_{q} \leq & \left\|\left(\mathcal{J}\left(2^{n}, x_{n}\right)\right)_{n}\right\|_{q}+\left\|\left(\mathcal{J}\left(2^{n}, v_{n}\right)\right)_{n}\right\|_{q} \\
\leq & 2+\left(\sum_{k \in \mathbb{Z}}\left(\max \left(\left\|u_{k-1}\right\|_{X_{0}}, 2^{k}\left\|u_{k-1}\right\|_{X_{1}}\right)\right)^{q}\right)^{1 / q} \\
& +2\left(\sum_{k \in \mathbb{Z}}\left(\max \left(\left\|u_{k}\right\|_{X_{0}}, 2^{k}\left\|u_{k}\right\|_{X_{1}}\right)\right)^{q}\right)^{1 / q} \\
\leq & 2+16 B
\end{aligned}
$$


Consequently, for $x \in N$, formula (2.6) implies that $\|x\|_{N_{\theta, q}} \leq 16 B+2$. Thus, the norms of $X_{\theta, q}$ and $N_{\theta, q}$ are equivalent on $N$.

We prove the converse under the condition $\beta_{\infty} \leq \alpha_{0}$. By assumption, there exists $D>0$ such that

$$
\|x\|_{N_{\theta, q}} \leq D\|x\|_{X_{\theta, q}}
$$

for all $x \in N$. By Lemma 2, it suffices to prove that the operator $T_{\theta}: l_{q}(\mu) \rightarrow \operatorname{Im} T_{\theta}$, $T_{\theta}=S-2^{\theta} I$, is an isomorphism.

Again, we distinguish two cases: a) the functional $\psi$ is not bounded on $X_{0} \cap X_{1}$ in the norm of $X_{\theta, q}$; b) $\psi$ is bounded on $X_{0} \cap X_{1}$ in the norm of $X_{\theta, q}$. In the second case, we let $\tilde{\psi} \in X_{\theta, q}{ }^{*}$ denote the extension of $\psi$.

So, let $a=\left(a_{n}\right) \in l_{q}(\mu),\|a\|_{l_{q}(\mu)} \leq 1$. As before, there exists a sequence $\left\{x_{n}\right\} \subset$ $X_{0} \cap X_{1}(n \in \mathbb{Z})$ with

$$
\mathcal{J}\left(2^{n}, x_{n}\right) \leq 2\left|a_{n}\right| \mu_{n}, \quad \psi\left(x_{n}\right)=a_{n} \quad(n \in \mathbb{Z}) .
$$

Therefore,

whence the series

$$
\left\|\left(\mathcal{J}\left(2^{n}, x_{n}\right)\right)_{n}\right\|_{q} \leq 2\|a\|_{l_{q}(\mu)} \leq 2
$$

$$
x=\sum_{k \in \mathbb{Z}} 2^{\theta k} x_{k}
$$

converges in $X_{0}+X_{1}, x \in X_{\theta, q}$, and $\|x\|_{X_{\theta, q}} \leq 2$. Moreover, in case b), we have $|\tilde{\psi}(x)| \leq$ $C\|x\|_{X_{\theta, q}} \leq 2 C$. Since the series in (2.8) converges in norm in $X_{\theta, q}$, we are led to the conclusion that

$$
\left|f_{\theta}(a)\right|=\left|\sum_{k \in \mathbb{Z}} 2^{\theta k} a_{k}\right|=\left|\sum_{k \in \mathbb{Z}} 2^{\theta k} \psi\left(x_{k}\right)\right|=|\tilde{\psi}(x)| \leq 2 C
$$

for every $a \in l_{q}(\mu)$ with $\|a\|_{l_{q}(\mu)} \leq 1$, i.e., $f_{\theta} \in l_{q}(\mu)^{*}$. By the facts proved above, $f_{\theta}(a)=0$ in this case, i.e., $x \in X_{\theta, q} \cap \tilde{N}$, where $\widetilde{N}:=\operatorname{Ker} \tilde{\psi}$.

In the next lemma, $Z_{\theta, q}$ is the closure of $N$ in $X_{\theta, q}$.

Lemma 5. We have $Z_{\theta, q}=X_{\theta, q}$ in case a) and $Z_{\theta, q}=X_{\theta, q} \cap \tilde{N}$ in case b).

Proof. If the functional $\psi$ is not bounded on $X_{0} \cap X_{1}$ in the norm of $X_{\theta, q}$, then $N$ is dense in $X_{0} \cap X_{1}$, and, consequently, also in $X_{\theta, q}$ (relative to the same norm); see [2, Theorem 3.4.2]. Therefore, $Z_{\theta, q}=X_{\theta, q}$.

In case b), if $x \in Z_{\theta, q}$, then $\tilde{\psi}(x)=0$, and, consequently, $Z_{\theta, q} \subset X_{\theta, q} \cap \tilde{N}$. To prove the reverse inclusion, take $x \in X_{\theta, q}$ with $\tilde{\psi}(x)=0$. There exists a sequence $\left\{x_{n}\right\} \subset X_{0} \cap X_{1}$ such that $x_{n} \rightarrow x$ in $X_{\theta, q}$. Since $\psi \in\left(X_{0} \cap X_{1}\right)^{*}$, we have $x_{n}=y_{n}+\lambda_{n} z$, where $y_{n} \in N$, $z \in X_{0} \cap X_{1}, \psi(z)=1$, and $\lambda_{n} \in \mathbb{R}$. Then $\psi\left(x_{n}\right)=\lambda_{n} \psi(z)=\lambda_{n} \rightarrow \tilde{\psi}(x)=0$. Therefore, $y_{n} \rightarrow x$ in $X_{\theta, q}$, whence $x \in Z_{\theta, q}$.

We continue the proof of the theorem. We remind the reader that the vector $x$ defined by (2.8) with $\psi\left(x_{k}\right)=a_{k}$ belongs to $X_{\theta, q}$ in case a) and to $X_{\theta, q} \cap \widetilde{N}$ in case b). In both cases, Lemma 5 implies the existence of a sequence $\left\{z_{n}\right\} \subset N$ such that

$$
z_{n} \rightarrow x \text { in } X_{\theta, q},\left\|z_{n+1}-z_{n}\right\|_{X_{\theta, q}} \leq 2^{-n}, \quad n=1,2, \ldots .
$$

It follows that

$$
x=\sum_{n=0}^{\infty}\left(z_{n+1}-z_{n}\right), \quad z_{0}=0,
$$


where the series converges absolutely in $X_{\theta, q}$. Since $\left\|z_{n+1}-z_{n}\right\|_{N_{\theta, q}} \leq 2^{-n} D$ by (2.7), we see that for every $n=0,1,2, \ldots$ there exists a sequence $\left\{z_{n, i}\right\}_{i \in \mathbb{Z}} \subset N$ with

$$
z_{n+1}-z_{n}=\sum_{i \in \mathbb{Z}} 2^{i \theta} z_{n, i}, \quad\left\|\left(\mathcal{J}\left(2^{i}, z_{n, i}\right)\right)_{i}\right\|_{q}<2^{1-n} D .
$$

Consequently,

$$
\begin{aligned}
\sum_{n=0}^{\infty}\left\|z_{n, i}\right\|_{X_{0} \cap X_{1}} & \leq \max \left(1,2^{-i}\right) \sum_{n=0}^{\infty} \mathcal{J}\left(2^{i}, z_{n, i}\right) \\
& \leq \max \left(1,2^{-i}\right) \sum_{n=0}^{\infty}\left\|\left(\mathcal{J}\left(2^{i}, z_{n, i}\right)\right)_{i}\right\|_{q} \\
& \leq 4 D \max \left(1,2^{-i}\right),
\end{aligned}
$$

and the series

$$
y_{i}=\sum_{n=0}^{\infty} z_{n, i}
$$

converges absolutely in $X_{0} \cap X_{1}$ for every $i \in \mathbb{Z}$. But $z_{n, i} \in N$, and $N$ is the kernel of the functional $\psi \in\left(X_{0} \cap X_{1}\right)^{*}$; therefore, $y_{i} \in N, i \in \mathbb{Z}$. Moreover, (2.11) and the Minkowski inequality show that

$$
\begin{aligned}
\left\|\left(\mathcal{J}\left(2^{i}, y_{i}\right)\right)_{i}\right\|_{q} & \leq\left\{\sum_{i \in \mathbb{Z}}\left(\sum_{n=0}^{\infty} \mathcal{J}\left(2^{i}, z_{n, i}\right)\right)^{q}\right\}^{1 / q} \\
& \leq \sum_{n=0}^{\infty}\left(\sum_{i \in \mathbb{Z}}\left(\mathcal{J}\left(2^{i}, z_{n, i}\right)\right)^{q}\right)^{1 / q} \leq 4 D .
\end{aligned}
$$

Therefore, the series $\sum_{i \in \mathbb{Z}} 2^{\theta i} y_{i}$ converges in $X_{0}+X_{1}$ to a vector $y \in X_{\theta, q}$. We show that $y=x$. By $(2.10)-(2.12)$, it suffices to prove that the double series

$$
\sum_{n=0}^{\infty} \sum_{i \in \mathbb{Z}} 2^{\theta i} z_{n, i}
$$

converges absolutely in $X_{0}+X_{1}$. To do this, we apply the Hölder inequality and (2.12), obtaining

$$
\begin{aligned}
\sum_{i=0}^{\infty} 2^{\theta i}\left\|z_{n, i}\right\|_{X_{1}} & \leq \sum_{i=0}^{\infty} \mathcal{J}\left(2^{i}, z_{n, i}\right) 2^{i(\theta-1)} \\
& \leq\left(\sum_{i=0}^{\infty} 2^{i(\theta-1) q^{\prime}}\right)^{1 / q^{\prime}}\left\|\left(\mathcal{J}\left(2^{i}, z_{n, i}\right)\right)_{i}\right\|_{q} \leq C_{\theta, q} 2^{1-n} D
\end{aligned}
$$

and

$$
\sum_{i=-\infty}^{-1} 2^{\theta i}\left\|z_{n, i}\right\|_{X_{0}} \leq\left(\sum_{i=-\infty}^{-1} 2^{i \theta q^{\prime}}\right)^{1 / q^{\prime}}\left\|\left(\mathcal{J}\left(2^{i}, z_{n, i}\right)\right)_{i}\right\|_{q} \leq C_{\theta, q}^{\prime} 2^{1-n} D
$$

whence

$$
\sum_{n=0}^{\infty} \sum_{i \in \mathbb{Z}} 2^{\theta i}\left\|z_{n, i}\right\|_{X_{0}+X_{1}} \leq 4 D \max \left(C_{\theta, q}, C_{\theta, q}^{\prime}\right)
$$

Thus, along with (2.8), both in case a) and in case b) we have a representation of the form

$$
x=\sum_{n \in \mathbb{Z}} 2^{\theta n} y_{n}, \quad \text { where } y_{n} \in N \text { and }\left\|\left(\mathcal{J}\left(2^{n}, y_{n}\right)\right)_{n}\right\|_{q} \leq 4 D
$$


Next, as in [3], consider the vectors

$$
u_{n}=x_{n}-y_{n} \text { and } v_{n}=\sum_{k=n+1}^{\infty} 2^{(k-n-1) \theta} u_{k} .
$$

Then

$$
\psi\left(u_{n}\right)=\psi\left(x_{n}\right)=a_{n}, \quad n \in \mathbb{Z},
$$

and from (2.8) and (2.13) it follows that

$$
\sum_{n \in \mathbb{Z}} 2^{\theta n} u_{n}=0, \quad\left\|\left(\mathcal{J}\left(2^{n}, u_{n}\right)\right)_{n}\right\|_{q} \leq 4 D+2 .
$$

Moreover,

$$
2^{n}\left\|v_{n}\right\|_{X_{1}} \leq \sum_{k=n+1}^{\infty} 2^{(k-n-1)(\theta-1)}\left\|u_{k}\right\|_{X_{1}} 2^{k}
$$

and by (2.15) we have

$$
\left\|v_{n}\right\|_{X_{0}} \leq\left\|\sum_{k=-\infty}^{n} 2^{(k-n-1) \theta} u_{k}\right\|_{X_{0}} \leq \sum_{k=-\infty}^{n} 2^{(k-n-1) \theta}\left\|u_{k}\right\|_{X_{0}} .
$$

Now, the elementary inequality $\max (a, b) \leq a+b(a, b>0)$ combined with the Minkowski inequality and (2.15) yields

$$
\begin{aligned}
& \left\|\left(\mathcal{J}\left(2^{n}, v_{n}\right)\right)_{n}\right\|_{q} \\
& \leq\left\{\sum_{n \in \mathbb{Z}}\left(\sum_{k=n+1}^{\infty} 2^{(k-n-1)(\theta-1)} \mathcal{J}\left(2^{k}, u_{k}\right)+\sum_{k=-\infty}^{n} 2^{(k-n-1) \theta} \mathcal{J}\left(2^{k}, u_{k}\right)\right)^{q}\right\}^{1 / q} \\
& \quad=\left\{\sum_{n \in \mathbb{Z}}\left(\sum_{l=0}^{\infty} 2^{l(\theta-1)} \mathcal{J}\left(2^{l+n+1}, u_{l+n+1}\right)+\sum_{l=-\infty}^{-1} 2^{l \theta} \mathcal{J}\left(2^{l+n+1}, u_{l+n+1}\right)\right)^{q}\right\}^{1 / q} \\
& \leq\left(\sum_{l=0}^{\infty} 2^{l(\theta-1)}+\sum_{l=-\infty}^{-1} 2^{l \theta}\right)\left\|\left(\mathcal{J}\left(2^{n}, u_{n}\right)\right)_{n}\right\|_{q} \leq C_{\theta}(4 D+2) .
\end{aligned}
$$

So, $v_{n} \in X_{0} \cap X_{1}, v=\sum_{n \in \mathbb{Z}} 2^{\theta n} v_{n} \in X_{\theta, q}$, and $\|v\|_{X_{\theta, q}} \leq C_{\theta}(4 D+2)$. By (0.6), we have $\psi\left(v_{n}\right) \leq \mu_{n}^{-1} \mathcal{J}\left(2^{n}, v_{n}\right)$. Consequently, the element $b=\left(\psi\left(v_{n}\right)\right)_{n}$ belongs to $l_{q}(\mu)$ and $\|b\|_{l_{q}(\mu)} \leq C_{\theta}(4 D+2)$. Furthermore, by (2.14) we have

$$
\begin{aligned}
\left(T_{\theta} b\right)_{n} & =b_{n-1}-2^{\theta} b_{n}=\psi\left(v_{n-1}\right)-2^{\theta} \psi\left(v_{n}\right) \\
& =\psi\left(\sum_{k=n}^{\infty} 2^{(k-n) \theta} u_{k}-\sum_{k=n+1}^{\infty} 2^{(k-n-1) \theta} u_{k}\right)=\psi\left(u_{n}\right)=a_{n}
\end{aligned}
$$

for all $n \in \mathbb{Z}$.

Thus, the operator $T_{\theta}: l_{q}(\mu) \rightarrow l_{q}(\mu)$ is closed. Moreover, $\operatorname{Im} T_{\theta}=l_{q}(\mu)$ in case a), and $\operatorname{Im} T_{\theta}=\operatorname{Ker} f_{\theta}$ in case b). Since $\beta_{\infty} \leq \alpha_{0}$ by assumption, Remark 1 implies that $T_{\theta}$ is an isomorphic embedding, and the theorem is proved.

Now, we present some corollaries. The first of them is a direct consequence of the proof of Theorem 1 .

Corollary 1. The following statements are equivalent:

1) the norms of $X_{\theta, q}$ and $N_{\theta, q}$ are equivalent on $N$, and $\psi$ extends up to a functional $\tilde{\psi} \in X_{\theta, q}{ }^{*}$

2) $\theta \in\left(\beta_{\infty}, \alpha_{0}\right)$. 
Corollary 2. Suppose a linear functional $\varphi$ is defined on some linear space $D \supset X_{0} \cap X_{1}$. Let $\psi$ be the restriction of $\varphi$ to $X_{0} \cap X_{1}$, and suppose that $\psi \in\left(X_{0} \cap X_{1}\right)^{*}, \psi \neq 0$. Let $\alpha, \beta, \alpha_{0}$, and $\beta_{\infty}$ be the dilation indices of the function $k(t)=\mathcal{K}\left(t, \psi ; X_{0}^{*}, X_{1}^{*}\right)$. We assume that $\beta_{\infty} \leq \alpha_{0}$. If

$$
\left(X_{0} \cap \operatorname{Ker} \varphi, X_{1} \cap \operatorname{Ker} \varphi\right)_{\theta, q}=\left(X_{0}, X_{1}\right)_{\theta, q} \cap \operatorname{Ker} \varphi,
$$

then (2.1) is true.

Proof. Relations (2.16) imply, in particular, that the norms of $\left(X_{0}, X_{1}\right)_{\theta, q}$ and $\left(X_{0} \cap\right.$ $\left.\operatorname{Ker} \varphi, X_{1} \cap \operatorname{Ker} \varphi\right)_{\theta, q}$ are equivalent on $N:=\operatorname{Ker} \psi$. On the other hand, the norms of $\left(X_{0} \cap \operatorname{Ker} \varphi, X_{1} \cap \operatorname{Ker} \varphi\right)_{\theta, q}$ and $\left(N_{0}, N_{1}\right)_{\theta, q}$ coincide on $N$. Therefore, the norms of $\left(X_{0}, X_{1}\right)_{\theta, q}$ and $\left(N_{0}, N_{1}\right)_{\theta, q}$ are equivalent on $N$, and Theorem 1 applies.

The last corollary is also a direct consequence of the proof of Theorem 1.

Corollary 3. Suppose $\psi \in\left(X_{0} \cap X_{1}\right)^{*}, \psi \neq 0$, and let $N=\operatorname{Ker} \psi$. If $\theta \in(0, \alpha) \cup(\beta, 1)$, then for every $x \in X_{\theta, q}$ there exists a representation

$$
x=\sum_{k=-\infty}^{\infty} 2^{\theta k} x_{k}, \quad x_{k} \in N
$$

with

$$
\left\|\left(\mathcal{J}\left(2^{k}, x_{k} ; X_{0}, X_{1}\right)\right)_{k}\right\|_{l_{q}} \leq C\|x\|_{X_{\theta, q}}
$$

where the constant $C>0$ does not depend on $x$. A similar statement is true also for $\theta \in\left(\beta_{\infty}, \alpha_{0}\right)$ if we replace $X_{\theta, q}$ with the intersection $X_{\theta, q} \cap \widetilde{N}$, where $\widetilde{N}$ is the kernel of the extension $\tilde{\psi}$ of $\psi$ to $X_{\theta, q}$.

Remark 2. Corollary 3 readily implies the results of [3] that guarantee the identity of the spaces $\left(X_{0}, X_{1}\right)_{\theta, q}$ and $\left(N_{0}, X_{1}\right)_{\theta, q}$. In that situation, $\psi \in X_{0}^{*}$ and $N_{0}:=\operatorname{Ker} \psi$ is endowed with the norm of $X_{0}$. First, we observe that presently $k(t):=\mathcal{K}\left(t, \psi ; X_{0}^{*}, X_{1}^{*}\right) \leq$ $C(t>0)$, whence $\alpha=\beta_{\infty}=0$. Next, $\left(X_{0}, X_{1}\right)_{\theta, q} \subset N_{0}+X_{1}$ because, if $x \in X_{\theta, q}$ and $x=x_{0}+x_{1}$ with $x_{i} \in X_{i}$, then also $x=y_{0}+y_{1}$, where $y_{0}=x_{0}-\psi\left(x_{0}\right) z \in N_{0}$, and $y_{1}=x_{1}+\psi\left(x_{0}\right) z \in X_{1}$; here $z$ is an element of $X_{0} \cap X$ satisfying $\psi(z)=1$. By Corollary 3 and the equivalence of the $\mathcal{K}$ - and $\mathcal{J}$-methods (see [2, Theorem 3.3.1]), we see that

$$
\left(N_{0}, X_{1}\right)_{\theta, q}=\left(X_{0}, X_{1}\right)_{\theta, q} \text { if } \theta \in(\beta, 1),
$$

and

$$
\left(N_{0}, X_{1}\right)_{\theta, q}=\left(X_{0}, X_{1}\right)_{\theta, q} \cap \tilde{N} \quad \text { if } \theta \in\left(0, \alpha_{0}\right),
$$

where $\widetilde{N}$ is the kernel of the extension $\tilde{\psi}$ of $\psi$ to $\left(X_{0}, X_{1}\right)_{\theta, q}$.

Theorem 1 implies in addition that $\left(N_{0}, X_{1}\right)_{\theta, q}$ is not closed in $\left(X_{0}, X_{1}\right)_{\theta, q}$ for $\theta \in$ $\left[\alpha_{0}, \beta\right]$.

Theorem 2. Suppose $\psi \in\left(X_{0} \cap X_{1}\right)^{*}, \psi \neq 0$. If $\theta \in\left(\beta_{\infty}, \alpha_{0}\right)$, then $\psi$ extends by continuity up to a functional $\tilde{\psi}_{\theta} \in\left(\left(X_{0}, X_{1}\right)_{\theta, q}\right)^{*}$. If $\theta \in(0, \alpha)$ (respectively, $\left.\theta \in(\beta, 1)\right)$, then $\psi$ extends by continuity up to a functional $\tilde{\psi}_{\theta} \in\left(\left(X_{0}, X_{1}\right)_{\theta, q} \cap X_{1}\right)^{*}$ (respectively, up to a functional $\left.\tilde{\psi}_{\theta} \in\left(\left(X_{0}, X_{1}\right)_{\theta, q} \cap X_{0}\right)^{*}\right)$. Moreover, if $\widetilde{N}_{\theta}:=\operatorname{Ker} \tilde{\psi}_{\theta}$, then in all three cases we have

$$
\left(X_{0} \cap \tilde{N}_{\theta}, X_{1} \cap \widetilde{N}_{\theta}\right)_{\theta, q}=\left(X_{0}, X_{1}\right)_{\theta, q} \cap \tilde{N}_{\theta} .
$$

Proof. If $\theta \in\left(\beta_{\infty}, \alpha_{0}\right)$, then $\psi$ extends up to $\tilde{\psi}_{\theta} \in\left(\left(X_{0}, X_{1}\right)_{\theta, q}\right)^{*}$ by Corollary 1 . We prove (2.17) in this case.

For $\widetilde{N}:=\widetilde{N}_{\theta}$, the inclusion

$$
\left(X_{0} \cap \tilde{N}, X_{1} \cap \tilde{N}\right)_{\theta, q} \subset\left(X_{0}, X_{1}\right)_{\theta, q} \cap \tilde{N}
$$


is obvious. So, by Corollary 3 , it suffices to prove the inclusion

$$
X_{\theta, q} \cap \widetilde{N} \subset X_{0} \cap \widetilde{N}+X_{1} \cap \widetilde{N} .
$$

First, we observe that

$$
X_{\theta, q} \subset X_{\theta, q} \cap X_{0}+X_{\theta, q} \cap X_{1} .
$$

Indeed, for every $x \in X_{\theta, q}$ there exists a sequence $\left\{x_{n}\right\} \subset X_{0} \cap X_{1}$ such that

$$
x=\sum_{k=-\infty}^{\infty} 2^{\theta k} x_{k} \text { (convergence in } X_{0}+X_{1} \text { ) }
$$

and $\left(\mathcal{J}\left(2^{k}, x_{k}\right)\right)_{k} \in l_{q}$. As before, it is easily seen that

$$
\sum_{n=-\infty}^{-1} 2^{\theta n} x_{n}=x_{0} \in X_{0} \text { (convergence in } X_{0} \text { ) }
$$

and

$$
\sum_{n=0}^{\infty} 2^{\theta n} x_{n}=x_{1} \in X_{1} \text { (convergence in } X_{1} \text { ). }
$$

At the same time, the definition of $X_{\theta, q}$ via the $\mathcal{J}$-method yields $x_{i} \in X_{\theta, q}(i=0,1)$, and (2.19) follows from the relation $x=x_{0}+x_{1}$.

Passing to the proof of (2.18), we take $z \in X_{0} \cap X_{1}$ with $\psi(z)=1$. If $x \in X_{\theta, q} \cap \tilde{N}$ and $x=x_{0}+x_{1}, x_{i} \in X_{\theta, q} \cap X_{i}(i=0,1)$, we put

$$
y_{0}=x_{0}+\tilde{\psi}_{\theta}\left(x_{1}\right) z, \quad y_{1}=x_{1}-\tilde{\psi}_{\theta}\left(x_{1}\right) z .
$$

Then $y_{i} \in X_{i} \cap \widetilde{N}(i=0,1)$, and (2.18) follows.

Now, let $\theta \in(0, \alpha) \cup(\beta, 1)$. If $\theta \in(0, \alpha)$, we use Lemmas 3 and 4 . For this, consider the Banach couple $\left(X_{0} \cap X_{1}, X_{1}\right)$ and the corresponding $\mathcal{K}$-functional

$$
k^{\prime}(t):=\mathcal{K}\left(t, \psi ;\left(X_{0} \cap X_{1}\right)^{*}, X_{1}^{*}\right)=\mathcal{K}\left(t, \psi ; X_{0}^{*}+X_{1}^{*}, X_{1}^{*}\right) .
$$

The dilation indices for the function $k^{\prime}(t)$ will be denoted by $\alpha^{\prime}, \beta^{\prime}, \alpha_{0}^{\prime}$, and $\beta_{\infty}^{\prime}$. Since $\psi \in\left(X_{0} \cap X_{1}\right)^{*}$, we have $k^{\prime}(t) \leq C(t>0)$ and, therefore, $\alpha^{\prime}=\beta_{\infty}^{\prime}=0$. Applying Lemma 3 to the couple $\left(X_{0}^{*}, X_{1}^{*}\right)$, we obtain $k^{\prime}(t) \asymp k(t):=\mathcal{K}\left(t, \psi ; X_{0}^{*}, X_{1}^{*}\right)$ for $t \in(0,1]$. So, the definition of the dilation indices implies $\alpha_{0}^{\prime} \geq \alpha$. Thus, $\theta \in\left(\beta_{\infty}^{\prime}, \alpha_{0}^{\prime}\right)$, and by the first statement of the theorem (which has already been proved) we see that $\psi$ extends by continuity up to a functional $\tilde{\psi}_{\theta} \in\left(\left(X_{0} \cap X_{1}, X_{1}\right)_{\theta, q}\right)^{*}$. But the latter space coincides with $\left(\left(X_{0}, X_{1}\right)_{\theta, q} \cap X_{1}\right)^{*}$ by Lemma 4 .

The case where $\theta \in(\beta, 1)$ reduces to the preceding case. We put

$$
k^{\prime \prime}(t):=\mathcal{K}\left(t, \psi ; X_{0}^{*},\left(X_{0} \cap X_{1}\right)^{*}\right),
$$

and denote by $\alpha^{\prime \prime}, \beta^{\prime \prime}, \alpha_{0}^{\prime \prime}$, and $\beta_{\infty}^{\prime \prime}$ the dilation indices for $k^{\prime \prime}$. Then $k^{\prime \prime}(t) \leq C t(t>0)$ and, therefore, $\alpha_{0}^{\prime \prime}=\beta^{\prime \prime}=1$. Next, we observe that

$$
k(t)=t l\left(\frac{1}{t}\right), \quad k^{\prime \prime}(t)=t m\left(\frac{1}{t}\right),
$$

where

$$
l(t):=\mathcal{K}\left(t, \psi ; X_{1}^{*}, X_{0}^{*}\right), \quad m(t):=\mathcal{K}\left(t, \psi ;\left(X_{0} \cap X_{1}\right)^{*}, X_{0}^{*}\right) .
$$

It is easily seen that

$$
\beta+\alpha^{l}=1, \quad \beta_{\infty}^{\prime \prime}+\alpha_{0}^{m}=1,
$$

where $\alpha^{l}$ and $\alpha_{0}^{m}$ stand for dilation indices of $l(t)$ and $m(t)$, respectively. From the above it is clear that $\alpha_{0}^{m} \geq \alpha^{l}$. But then (2.21) immediately implies the inequality $\beta_{\infty}^{\prime \prime} \leq \beta$. 
Thus, if $\theta \in(\beta, 1)$, then $\theta \in\left(\beta_{\infty}^{\prime \prime}, \alpha_{0}^{\prime \prime}\right)$, and again Lemma 4 shows that $\psi$ extends up to a functional $\tilde{\psi}_{\theta} \in\left(\left(X_{0}, X_{1}\right)_{\theta, q} \cap X_{0}\right)^{*}$.

It remains to prove $(2.17)$ for $\theta \in(0, \alpha)$ or $\theta \in(\beta, 1)$. For definiteness, let $\theta \in(0, \alpha)$ (the other case is treated similarly). By Corollary 3 , it again suffices to prove (2.18), in which $\widetilde{N}:=\widetilde{N}_{\theta}$ is the kernel of the extension $\tilde{\psi}_{\theta}$ of $\psi$.

If $x \in X_{\theta, q} \cap \tilde{N}$, we use (2.19) to represent $x$ in the form $x=x_{0}+x_{1}$, where $x_{i} \in$ $X_{\theta, q} \cap X_{i}(i=0,1)$. It follows that $x_{1}$ and, with it, $x_{0}$ belong to the domain of $\tilde{\psi}_{\theta}$. Consequently, defining $y_{0}$ and $y_{1}$ by (2.20), we obtain $x=y_{0}+y_{1}$, where $y_{i} \in X_{i} \cap \widetilde{N}$ $(i=0,1)$. Thus, we arrive at $(2.18)$ and, therefore, also at $(2.17)$.

Corollary 4. Suppose a linear functional $\varphi$ is defined on a linear space $D, D \supset X_{0} \cap X_{1}$, the restriction $\psi$ of $\varphi$ to $X_{0} \cap X_{1}$ is a bounded functional on $X_{0} \cap X_{1}$, and $\psi \neq 0$. For every $\theta$ satisfying $(2.1)$, let $\tilde{\psi}_{\theta}$ denote a continuous extension of $\psi$ with domain $\widetilde{M}_{\theta}$ (equal either to $X_{\theta, q}$, or to $X_{\theta, q} \cap X_{1}$, or to $\left.X_{\theta, q} \cap X_{0}\right)$, as in Theorem 2. If $\widetilde{M}_{\theta} \subset D$ and $\varphi(x)=\tilde{\psi}_{\theta}(x)$ for $x \in \widetilde{M}_{\theta}$, then (2.16) is fulfilled.

Proof. If $\theta \in(0, \alpha) \cup(\beta, 1)$, then, again by Corollary 3 , it suffices to show that

$$
X_{\theta, q} \cap \operatorname{Ker} \varphi \subset X_{0} \cap \operatorname{Ker} \varphi+X_{1} \cap \operatorname{Ker} \varphi .
$$

Let $\theta \in(0, \alpha)$ for definiteness. If $x \in X_{\theta, q} \cap \operatorname{Ker} \varphi$, then (2.19) shows that $x=x_{0}+x_{1}$, where $x_{i} \in X_{\theta, q} \cap X_{i}(i=0,1)$. Then $x_{1} \in \widetilde{M}_{\theta}:=X_{\theta, q} \cap X_{1}$. Defining $y_{0}$ and $y_{1}$ by (2.20), we obtain $x=y_{0}+y_{1}$; hence $y_{1}$ and, with it, $y_{0}$ belong to $\operatorname{Ker} \varphi$. Thus, (2.22) is proved. The case of $\theta \in(\beta, 1)$ is treated similarly.

Now, let $\theta \in\left(\beta_{\infty}, \alpha_{0}\right)$. If $X_{\theta, q} \cap \operatorname{Ker} \varphi=X_{\theta, q} \cap \widetilde{N}_{\theta}$, where $\widetilde{N}_{\theta}:=\operatorname{Ker} \tilde{\psi}_{\theta}$, then the

result follows from Theorem 2 (or Corollary 3). Suppose $X_{\theta, q} \cap \operatorname{Ker} \varphi \stackrel{\neq}{\supset} X_{\theta, q} \cap \widetilde{N}_{\theta}$. Since $\widetilde{N}_{\theta}$ is a subspace of codimension 1 in $X_{\theta, q}$, we have $X_{\theta, q} \subset \operatorname{Ker} \varphi$, i.e., $\varphi(x)=0$ for all $x \in X_{\theta, q}$. This contradicts the assumptions of the corollary.

\section{$\S 3$. The $\mathcal{K}$-Functional on Couples of intersections}

Let $\left(X_{0}, X_{1}\right)$ be a Banach couple with $X_{0} \cap X_{1}$ dense in $X_{0}$ and in $X_{1}$, and let $Y_{0}$ be a closed subspace of codimension 1 in $X_{0}$. In 8 a formula connecting $\mathcal{K}\left(t, x ; Y_{0}, X_{1}\right)$ and $\mathcal{K}\left(t, x ; X_{0}, X_{1}\right)$ was found (see also [12, Theorem 2.1]). We show that, under a certain additional assumption, a similar result holds true also for a normed couple of intersections generated by a linear functional.

Let $\psi$ be a linear functional defined on a subspace $M \supset X_{0} \cap X_{1}$, and let $N=\operatorname{Ker} \psi$. Moreover, we assume that the restriction of $\psi$ to $X_{0} \cap X_{1}$ is a bounded nonzero functional on this space. We put $Y_{i}=X_{i} \cap N(i=0,1)$.

For every $y \in Y_{0}+Y_{1}$ and every $t>0$, there exists an "optimal" decomposition

$$
y=y_{0}(t)+y_{1}(t), \quad y_{i}(t) \in X_{i}, \quad i=0,1,
$$

with the property

$$
\frac{1}{2}\left(\left\|y_{0}(t)\right\|_{X_{0}}+t\left\|y_{1}(t)\right\|_{X_{1}}\right) \leq \mathcal{K}\left(t, y ; X_{0}, X_{1}\right) \leq\left\|y_{0}(t)\right\|_{X_{0}}+t\left\|y_{1}(t)\right\|_{X_{1}} .
$$

Theorem 3. Suppose $X_{0} \cap X_{1}$ is dense both in $X_{0}$ and in $X_{1}$, and for every $y \in Y_{0}+Y_{1}$ an "optimal" decomposition (3.1) can be chosen in such a way that $y_{0}(t) \in M$ for all $t>0$. Then

$$
\mathcal{K}\left(t, y ; Y_{0}, Y_{1}\right) \asymp \mathcal{K}\left(t, y ; X_{0}, X_{1}\right)+\frac{\left|\psi\left(y_{0}(t)\right)\right|}{\mathcal{K}\left(t^{-1}, \psi ; X_{0}^{*}, X_{1}^{*}\right)},
$$

where the constants of equivalence do not depend on $y \in Y_{0}+Y_{1}$ and $t>0$. 
Proof. Take $h \in X_{0} \cap X_{1}$ with $\psi(h) \neq 0$. For $y \in Y_{0}+Y_{1}$, consider a decomposition (3.1) satisfying the assumptions of the theorem. Putting

$$
z_{0}(t)=y_{0}(t)-\frac{\psi\left(y_{0}(t)\right) h}{\psi(h)}, \quad z_{1}(t)=y_{1}(t)+\frac{\psi\left(y_{0}(t)\right) h}{\psi(h)},
$$

we see that $z_{i}(t) \in Y_{i}$ and $y=z_{0}(t)+z_{1}(t)$. Consequently,

$$
\begin{aligned}
\mathcal{K}\left(t, y ; Y_{0}, Y_{1}\right) & \leq\left\|z_{0}(t)\right\|_{X_{0}}+t\left\|z_{1}(t)\right\|_{X_{1}} \\
& \leq\left\|y_{0}(t)\right\|_{X_{0}}+t\left\|y_{1}(t)\right\|_{X_{1}}+\left|\psi\left(y_{0}(t)\right)\right| \frac{\|h\|_{X_{0}}+t\|h\|_{X_{1}}}{|\psi(h)|} \\
& \leq 2 \mathcal{K}\left(t, y ; X_{0}, X_{1}\right)+2\left|\psi\left(y_{0}(t)\right)\right| \frac{\mathcal{J}\left(t, h ; X_{0}, X_{1}\right)}{|\psi(h)|}
\end{aligned}
$$

by (3.2). Applying (0.6) and passing to the infimum over all $h \in X_{0} \cap X_{1}$ with $\psi(h) \neq 0$ in the second term on the right, we obtain

$$
\mathcal{K}\left(t, y ; Y_{0}, Y_{1}\right) \leq 2\left(\mathcal{K}\left(t, y ; X_{0}, X_{1}\right)+\frac{\left|\psi\left(y_{0}(t)\right)\right|}{\mathcal{K}\left(t^{-1}, \psi ; X_{0}^{*}, X_{1}^{*}\right)}\right) .
$$

To prove the reverse inequality, if suffices to show that

$$
\frac{\left|\psi\left(y_{0}(t)\right)\right|}{\mathcal{K}\left(t^{-1}, \psi ; X_{0}^{*}, X_{1}^{*}\right)} \leq 4 \mathcal{K}\left(t, y ; Y_{0}, Y_{1}\right)
$$

We may assume that $\psi\left(y_{0}(t)\right) \neq 0$ (otherwise there is nothing to prove). For $t>0$, we find a decomposition $y=z_{0}(t)+z_{1}(t), z_{i}(t) \in Y_{i}$, such that

$$
\mathcal{K}\left(t, y ; Y_{0}, Y_{1}\right) \geq \frac{1}{2}\left(\left\|z_{0}(t)\right\|_{X_{0}}+t\left\|z_{1}(t)\right\|_{X_{1}}\right)
$$

(an "optimal" decomposition of $y$ in the couple $\left(Y_{0}, Y_{1}\right)$ ). Using this and also the decomposition (3.1), we define $h=y_{0}(t)-z_{0}(t)=z_{1}(t)-y_{1}(t)$. Then $h \in X_{0} \cap X_{1}$, and the relation $z_{0}(t) \in Y_{0} \subset N$ implies that

$$
\psi(h)=\psi\left(y_{0}(t)\right)-\psi\left(z_{0}(t)\right)=\psi\left(y_{0}(t)\right) \neq 0 .
$$

Therefore,

$$
z_{0}(t)=y_{0}(t)-\frac{\psi\left(y_{0}(t)\right) h}{\psi(h)}, \quad z_{1}(t)=y_{1}(t)+\frac{\psi\left(y_{0}(t)\right) h}{\psi(h)},
$$

and by (3.4), (3.2), and (0.6) we obtain

$$
\begin{aligned}
\mathcal{K}\left(t, y ; Y_{0}, Y_{1}\right) & \geq \frac{1}{2}\left(\left\|y_{0}(t)-\frac{\psi\left(y_{0}(t)\right)}{\psi(h)} h\right\|_{X_{0}}+t\left\|y_{1}(t)+\frac{\psi\left(y_{0}(t)\right)}{\psi(h)} h\right\|_{X_{1}}\right) \\
& \geq \frac{1}{2}\left(\left|\psi\left(y_{0}(t)\right)\right| \frac{\|h\|_{X_{0}}+t\|h\|_{X_{1}}}{|\psi(h)|}-\left(\left\|y_{0}(t)\right\|_{X_{0}}+t\left\|y_{1}(t)\right\|_{X_{1}}\right)\right) \\
& \geq \frac{1}{2}\left|\psi\left(y_{0}(t)\right)\right| \inf _{h \in X_{0} \cap X_{1}, \psi(h) \neq 0} \frac{\mathcal{J}\left(t, \psi ; X_{0}, X_{1}\right)}{|\psi(h)|}-\mathcal{K}\left(t, y ; X_{0}, X_{1}\right) \\
& =\frac{1}{2} \frac{\left|\psi\left(y_{0}(t)\right)\right|}{\mathcal{K}\left(t^{-1}, \psi ; X_{0}^{*}, X_{1}^{*}\right)}-\mathcal{K}\left(t, y ; X_{0}, X_{1}\right) .
\end{aligned}
$$

Now, (3.4) follows immediately, because $\mathcal{K}\left(t, y ; X_{0}, X_{1}\right) \leq \mathcal{K}\left(t, y ; Y_{0}, Y_{1}\right)$. 


\section{§4. Interpolation of INTERSECtions of WEighted $L_{p}$-SPACES GENERATED BY AN INTEGRAL FUNCTIONAL}

As usual, for a positive measurable function $w(x)$ on $(0, \infty)$, we define $L_{p}(w)$ as the space of measurable functions $f:(0, \infty) \rightarrow \mathbb{R}$ with the norm

$$
\|f\|_{w, p}=\left(\int_{0}^{\infty}|f(x)|^{p} w(x) d x\right)^{1 / p} \quad \text { if } 1 \leq p<\infty,
$$

and

$$
\|f\|_{w, \infty}=\underset{x>0}{\operatorname{ess} \sup }(|f(x)| w(x)) .
$$

For an arbitrary measurable function $g:(0, \infty) \rightarrow \mathbb{R}$, consider the linear functional

$$
\varphi_{g}(f)=\int_{0}^{\infty} f(x) g(x) d x
$$

with the domain $D_{g}$ consisting of all measurable functions $f:(0, \infty) \rightarrow \mathbb{R}$ such that $\int_{a}^{b}|f(x) g(x)| d x<\infty$ for all $0<a<b<\infty$ and the following limit exists:

$$
\lim _{a \rightarrow 0, b \rightarrow \infty} \int_{a}^{b} f(x) g(x) d x .
$$

Basically, we shall be interested in the case where $g(x) \equiv 1$. Then we use the symbols $\varphi$ and $D$, respectively.

First, we find conditions ensuring the relation

$$
\left(L_{p_{0}}\left(w_{0}\right) \cap \operatorname{Ker} \varphi, L_{p_{1}}\left(w_{1}\right) \cap \operatorname{Ker} \varphi\right)_{\theta, q}=\left(L_{p_{0}}\left(w_{0}\right), L_{p_{1}}\left(w_{1}\right)\right)_{\theta, q} \cap \operatorname{Ker} \varphi .
$$

Thus, $g(x) \equiv 1$, and we consider three cases distinguished in terms of $p_{0}$ and $p_{1}$.

a) $1 \leq p_{0}<p_{1}<\infty$. We denote by $\psi$ the restriction of the functional $\varphi(f)=$ $\int_{0}^{\infty} f(x) d x$ to $L_{p_{0}}\left(w_{0}\right) \cap L_{p_{1}}\left(w_{1}\right)$. For every Banach couple $\left(X_{0}, X_{1}\right)$ with $X_{0} \cap X_{1}$ dense both in $X_{0}$ and in $X_{1}$, we have $\left(X_{0} \cap X_{1}\right)^{*}=X_{0}^{*}+X_{1}^{*}$ isometrically (see [2, §2.7]). Thus, if $\frac{1}{p_{i}^{\prime}}+\frac{1}{p_{i}}=1(i=0,1)$, then the condition $\psi \in\left(L_{p_{0}}\left(w_{0}\right) \cap L_{p_{1}}\left(w_{1}\right)\right)^{*}$ is equivalent to the condition

or

$$
1 \in L_{p_{0}^{\prime}}\left(w_{0}^{-1 /\left(p_{0}-1\right)}\right)+L_{p_{1}^{\prime}}\left(w_{1}^{-1 /\left(p_{1}-1\right)}\right) \quad\left(p_{0}>1\right)
$$

$1 \in L_{\infty}\left(w_{0}^{-1}\right)+L_{p_{1}^{\prime}}\left(w_{1}^{-1 /\left(p_{1}-1\right)}\right) \quad\left(p_{0}=1\right)$.

In its turn, this is equivalent to the finiteness of the function

$$
k(t)=\mathcal{K}\left(t, 1 ; L_{p_{0}^{\prime}}\left(w_{0}^{-1 /\left(p_{0}-1\right)}\right), L_{p_{1}^{\prime}}\left(w_{1}^{-1 /\left(p_{1}-1\right)}\right)\right) \quad\left(p_{0}>1\right)
$$

or

$$
k^{\prime}(t)=\mathcal{K}\left(t, 1 ; L_{\infty}\left(w_{0}^{-1}\right), L_{p_{1}^{\prime}}\left(w_{1}^{-1 /\left(p_{1}-1\right)}\right)\right) \quad\left(p_{0}=1\right)
$$

for some (all) $t>0$.

Assume first that $p_{0}>1$. After reduction to one weight function, we can write

$$
k(t)=\mathcal{K}\left(t, u ; L_{p_{0}^{\prime}}(v), L_{p_{1}^{\prime}}(v)\right),
$$

where

$$
u(x)=w_{0}(x)^{-\frac{p_{1}-1}{p_{1}-p_{0}}} w_{1}(x)^{\frac{p_{0}-1}{p_{1}-p_{0}}}, \quad v(x)=w_{0}(x)^{\frac{p_{1}}{p_{1}-p_{0}}} w_{1}(x)^{-\frac{p_{0}}{p_{1}-p_{0}}} .
$$

Applying the Holmstedt formula (see [2, Subsection 3.6]), we obtain

$$
\begin{aligned}
k(t) & =t \mathcal{K}\left(\frac{1}{t}, u ; L_{p_{1}^{\prime}}(v), L_{p_{0}^{\prime}}(v)\right) \\
& \asymp t\left(\int_{0}^{t^{-r}} u^{*}(s)^{p_{1}^{\prime}} d s\right)^{1 / p_{1}^{\prime}}+\left(\int_{t^{-r}}^{\infty} u^{*}(s)^{p_{0}^{\prime}} d s\right)^{1 / p_{0}^{\prime}},
\end{aligned}
$$


where $\frac{1}{r}=\frac{1}{p_{0}}-\frac{1}{p_{1}}$, and $u^{*}(s)$ is a nonincreasing rearrangement of $u(x)$ with respect to the measure $\nu(E)=\int_{E} v(x) d x$. Putting

$$
F_{t}=\left\{x>0: u(x)>u^{*}\left(t^{-r}\right)\right\}, \quad F_{t}^{c}=(0, \infty) \backslash F_{t},
$$

we have $k(t) \asymp k_{1}(t)$, where

$$
k_{1}(t)=t\left(\int_{F_{t}} w_{1}(x)^{-1 /\left(p_{1}-1\right)} d x\right)^{1 / p_{1}^{\prime}}+\left(\int_{F_{t}^{c}} w_{0}(x)^{-1 /\left(p_{0}-1\right)} d x\right)^{1 / p_{0}^{\prime}}
$$

Much in the same way, it can be shown that for $p_{0}=1$ we have $k^{\prime}(t) \asymp k_{1}^{\prime}(t)$, where

$$
k_{1}^{\prime}(t)=t\left(\int_{F_{t}} w_{1}(x)^{-1 /\left(p_{1}-1\right)} d x\right)^{1 / p_{1}^{\prime}} .
$$

We also note that $r=p_{1}^{\prime}$ and $u(x)=w_{0}^{-1}(x)$ in the latter case.

Theorem 4. Suppose $1 \leq p_{0}<p_{1}<\infty$, and let the functions $u(x)$ and $v(x)$ and the sets $F_{t}, F_{t}^{c}(t>0)$ be defined by (4.2) and (4.3), respectively. Suppose the function

$$
k_{1}(t)=t\left(\int_{F_{t}} w_{1}(x)^{-1 /\left(p_{1}-1\right)} d x\right)^{1 / p_{1}^{\prime}}+\left(\int_{F_{t}^{c}} w_{0}(x)^{-1 /\left(p_{0}-1\right)} d x\right)^{1 / p_{0}^{\prime}}
$$

(if $p_{0}=1$, the second term on the right should be suppressed) is finite for some (all) $t>0$, and let $\alpha, \beta, \alpha_{0}$, and $\beta_{\infty}$ denote its dilation indices. If $\beta_{\infty} \leq \alpha_{0}, 0<\theta<1$, and $1 \leq q<\infty$, then (4.1) is true if and only if (2.1) is true.

Proof. If (4.1) is fulfilled, then Corollary 2 is applicable by the assumptions of the theorem, and we obtain $(2.1)$.

Conversely, let (2.1) be fulfilled. By the arguments preceding the theorem, the restriction $\psi$ of $\varphi$ to $L_{p_{0}}\left(w_{0}\right) \cap L_{p_{1}}\left(w_{1}\right)$ belongs to $\left(L_{p_{0}}\left(w_{0}\right) \cap L_{p_{1}}\left(w_{1}\right)\right)^{*}$. Moreover, $\psi \neq 0$. Consequently, by Theorem 2, there is a continuous extension of $\psi$ up to a functional $\tilde{\psi}_{\theta} \in \widetilde{M}_{\theta}^{*}$, where $\widetilde{M}_{\theta}$ is either $\left(L_{p_{0}}\left(w_{0}\right), L_{p_{1}}\left(w_{1}\right)\right)_{\theta, q}$, or $\left(L_{p_{0}}\left(w_{0}\right), L_{p_{1}}\left(w_{1}\right)\right)_{\theta, q} \cap L_{p_{1}}\left(w_{1}\right)$, or $\left(L_{p_{0}}\left(w_{0}\right), L_{p_{1}}\left(w_{1}\right)\right)_{\theta, q} \cap L_{p_{0}}\left(w_{0}\right)$, depending on whether $\theta$ belongs to $\left(\beta_{\infty}, \alpha_{0}\right),(0, \alpha)$, or $(\beta, 1)$.

We show that $\widetilde{M}_{\theta} \subset D$ for every $\theta \in(0, \alpha) \cup\left(\beta_{\infty}, \alpha_{0}\right) \cup(\beta, 1)$, and for $f \in \widetilde{M}_{\theta}$ we have

$$
\tilde{\psi}_{\theta}(f)=\varphi(f)=\int_{0}^{\infty} f(x) d x .
$$

First, $L_{p_{0}}\left(w_{0}\right) \cap L_{p_{1}}\left(w_{1}\right) \subset D$ by assumption, and for $f \in L_{p_{0}}\left(w_{0}\right) \cap L_{p_{1}}\left(w_{1}\right)$ we have the estimate

$$
\int_{0}^{\infty}|f(x)| d x \leq C_{\theta}\|f\|_{\widetilde{M}_{\theta}} .
$$

Now, let $f \in \widetilde{M}_{\theta}$, and let a sequence $\left\{f_{n}\right\} \subset L_{p_{0}}\left(w_{0}\right) \cap L_{p_{1}}\left(w_{1}\right)$ satisfy $\left\|f_{n}-f\right\|_{\widetilde{M}_{\theta}} \rightarrow 0$ as $n \rightarrow \infty$. Then $f_{n} \chi_{[0, b]} \rightarrow f \chi_{[0, b]}$ as $n \rightarrow \infty$ in measure for every $b>0$ (here and below $\chi_{F}$ stands for the characteristic function of a set $\left.F \subset \mathbb{R}\right)$. By (4.5) we obtain

$$
\int_{0}^{b}\left|f_{n}(x)\right| d x \leq C_{\theta}\left\|f_{n}\right\|_{\widetilde{M}_{\theta}}
$$

and the Fatou lemma shows that

$$
\int_{0}^{\infty}|f(x)| d x \leq C_{\theta} \lim _{n \rightarrow \infty}\left\|f_{n}\right\|_{\widetilde{M}_{\theta}}=C_{\theta}\|f\|_{\widetilde{M}_{\theta}},
$$


i.e., $\widetilde{M}_{\theta} \subset L_{1}(0, \infty) \subset D$. Next, applying (4.5) once again, we see that

$$
\int_{0}^{\infty}\left|f_{n}(x)-f_{m}(x)\right| d x \leq C_{\theta}\left\|f_{n}-f_{m}\right\|_{\widetilde{M}_{\theta}} \quad(n, m=1,2, \ldots) .
$$

Consequently, the sequence $\left\{f_{n}\right\}$ has a limit in $L_{1}(0, \infty)$, which must coincide with $f$. Since

$$
\int_{0}^{\infty} f_{n}(x) d x=\tilde{\psi}_{\theta}\left(f_{n}\right) \rightarrow \tilde{\psi}_{\theta}(f)
$$

at the same time, we arrive at (4.4). Now, all conditions of Corollary 4 are satisfied. Applying this corollary, we obtain (4.1).

If the numbers $p_{0}, p_{1}$ and the weight functions $w_{0}(x)$ and $w_{1}(x)$ satisfy some additional conditions, we can obtain more specific results. First, let $p_{0}>1$, and assume that

$(*) w_{0}(x)$ is monotone increasing, $w_{0}(2 x) \leq C_{1} w_{0}(x)(x>0)$, and

$$
\int_{x}^{\infty} w_{0}(s)^{\frac{1}{1-p_{0}}} d s \leq C_{2} x w_{0}(x)^{\frac{1}{1-p_{0}}}, \quad x>0 ;
$$

next, either $w_{1}(x)$ is monotone decreasing and $w_{1}(x) \leq C_{3} w_{1}(2 x)(x>0)$, or both $w_{1}(x)$ and $w(x)=\frac{w_{0}(x)}{w_{1}(x)}$ are monotone increasing and

$$
\int_{0}^{x} w_{1}(s)^{\frac{1}{1-p_{1}}} d s \leq C_{4} x w_{1}(x)^{\frac{1}{1-p_{1}}} \quad(x>0) .
$$

It is easily seen that, under condition $(*), u(x)$ is monotone decreasing and $v(x)$ is monotone increasing. In particular, it follows that $F_{t}=(0, s(t))$, where $s(t)$ is a solution of the equation

$$
V(x):=\int_{0}^{x} v(s) d s=t^{-r}, \quad \frac{1}{r}=\frac{1}{p_{0}}-\frac{1}{p_{1}} .
$$

Moreover, it is easily seen that $V(x) \asymp x v(x)$. Therefore,

$$
s(t) v(s(t)) \asymp t^{-r} .
$$

Now, on the one hand, we have

$$
k_{1}(t) \geq\left(\int_{s(t)}^{2 s(t)} w_{0}(x)^{-1 /\left(p_{0}-1\right)} d x\right)^{1 / p_{0}^{\prime}} \geq C_{1}^{-1 / p_{0}} s(t)^{1 / p_{0}^{\prime}} w_{0}(s(t))^{-1 / p_{0}}
$$

by $(*)$. On the other hand, $(*)$ and (4.7) imply that

$$
\begin{aligned}
k_{1}(t) & \leq C\left(s(t)^{1 / p_{0}^{\prime}} w_{0}(s(t))^{-1 / p_{0}}+t s(t)^{1 / p_{1}^{\prime}} w_{1}(s(t))^{-1 / p_{1}}\right) \\
& =C s(t)^{1 / p_{0}^{\prime}} w_{0}(s(t))^{-1 / p_{0}}\left(1+t s(t)^{1 / r} v(s(t))^{1 / r}\right) \\
& \leq C^{\prime} s(t)^{1 / p_{0}^{\prime}} w_{0}(s(t))^{-1 / p_{0}} .
\end{aligned}
$$

Thus, under condition $(*)$ we have the equivalence

$$
k_{1}(t) \asymp s(t)^{1 / p_{0}^{\prime}} w_{0}(s(t))^{-1 / p_{0}} .
$$

If $p_{0}=1$, a slightly less restrictive condition can be employed:

$(* *) w_{0}(x)$ is monotone increasing and $w_{0}(2 x) \leq C_{1} w_{0}(x)(x>0)$; next, either $w_{1}(x)$ is monotone decreasing and $w_{1}(x) \leq C_{2} w_{1}(2 x)(x>0)$, or both $w_{1}(x)$ and $w(x)=\frac{w_{0}(x)}{w_{1}(x)}$ are monotone increasing and

$$
\int_{0}^{x} w_{1}(s)^{\frac{1}{1-p_{1}}} d s \leq C_{3} x w_{1}(x)^{\frac{1}{1-p_{1}}} \quad(x>0) .
$$


Much as in the case where $p_{0}>1$, it can be shown that $(* *)$ implies

$$
k_{1}^{\prime}(t) \asymp w_{0}(s(t))^{-1} .
$$

As a result, applying Theorem 4, we arrive at the following statement.

Corollary 5. Suppose two numbers $p_{0}, p_{1}\left(1 \leq p_{0}<p_{1}<\infty\right)$ and two weight functions $w_{0}(x), w_{1}(x)$ satisfy $(*)$ if $p_{0}>1$ and satisfy $(* *)$ if $p_{0}=1$. Let $\alpha, \beta, \alpha_{0}$, and $\beta_{\infty}$ be the dilation indices of the function $k_{2}(t)=s(t)^{\frac{p_{0}-1}{p_{0}}} w_{0}(s(t))^{-\frac{1}{p_{0}}}$, where $s(t)$ is the solution of equation (4.6). If $\beta_{\infty} \leq \alpha_{0}, 0<\theta<1$, and $1 \leq q<\infty$, then (4.1) is equivalent to (2.1).

b) $1<p_{0}=p_{1}=p<\infty$. It is well known (see [2, Subsection 5.4]) that in this case

$$
\begin{aligned}
k(t) & =\mathcal{K}\left(t, 1 ; L_{p^{\prime}}\left(w_{0}^{-1 /(p-1)}\right), L_{p^{\prime}}\left(w_{1}^{-1 /(p-1)}\right)\right) \\
& \asymp\left(\int_{0}^{\infty} \min \left(w_{0}(x)^{-1 /(p-1)}, t^{p^{\prime}} w_{1}(x)^{-1 /(p-1)}\right) d x\right)^{1 / p^{\prime}} \\
& =\left(t^{p^{\prime}} \int_{F_{t}} w_{1}(x)^{-1 /(p-1)} d x+\int_{F_{t}^{c}} w_{0}(x)^{-1 /(p-1)} d x\right)^{1 / p^{\prime}},
\end{aligned}
$$

where

$$
F_{t}=\left\{x>0: \frac{w_{0}(x)}{w_{1}(x)}<t^{-p}\right\}, \quad F_{t}^{c}=(0, \infty) \backslash F_{t} .
$$

The following statement is proved much as Theorem 4 .

Theorem 5. Let $p \in(1, \infty)$. Suppose $w_{0}(x)$ and $w_{1}(x)$ are such that the function

$$
k_{3}(t)=\left(t^{p^{\prime}} \int_{F_{t}} w_{1}(x)^{-1 /(p-1)} d x+\int_{F_{t}^{c}} w_{0}(x)^{-1 /(p-1)} d x\right)^{1 / p^{\prime}},
$$

where $F_{t}$ and $F_{t}^{c}$ are defined by (4.8), is finite for some (all) $t>0$. If $\alpha, \beta, \alpha_{0}$, and $\beta_{\infty}$ are the dilation indices of the function $k_{3}(t)$ and $\beta_{\infty} \leq \alpha_{0}$, then the relation

$$
\left(L_{p}\left(w_{0}\right) \cap \operatorname{Ker} \varphi, L_{p}\left(w_{1}\right) \cap \operatorname{Ker} \varphi\right)_{\theta, q}=\left(L_{p}\left(w_{0}\right), L_{p}\left(w_{1}\right)\right)_{\theta, q} \cap \operatorname{Ker} \varphi
$$

is equivalent to (2.1).

Suppose $(*)$ is fulfilled for $p_{0}=p_{1}=p, w_{0}(x)$, and $w_{1}(x)$. Then $F_{t}=\left(0, w^{-1}\left(t^{-p}\right)\right)$, where $w^{-1}(x)$ is the function inverse to $w(x)=w_{0}(x) / w_{1}(x)$. Therefore, arguing as we did before Corollary 5 , we obtain yet another statement.

Corollary 6. Suppose $(*)$ is fulfilled for $p_{0}=p_{1}=p \in(1, \infty), w_{0}(x)$, and $w_{1}(x)$. Let $\alpha, \beta, \alpha_{0}$, and $\beta_{\infty}$ be the dilation indices of the function

$$
k_{4}(t)=\left(w^{-1}\left(t^{-p}\right)\right)^{(p-1) / p}\left(w_{0}\left(w^{-1}\left(t^{-p}\right)\right)\right)^{-1 / p},
$$

and let $\beta_{\infty} \leq \alpha_{0}$. Then (4.9) is equivalent to (2.1).

c) $p_{0}=p_{1}=1$. In this case

$$
k(t)=\mathcal{K}\left(t, 1 ; L_{\infty}\left(1 / w_{0}\right), L_{\infty}\left(1 / w_{1}\right)\right)=\sup _{x>0} \frac{1}{w_{0}(x)} \min \left(1, t \frac{w_{0}(x)}{w_{1}(x)}\right) .
$$

If the weight functions obey the condition

$w_{0}(x)$ is monotone increasing, $w_{1}(x)$ is monotone decreasing, and

$$
\lim _{x \rightarrow 0} w(x)=\lim _{x \rightarrow \infty} \frac{1}{w(x)}=0
$$

then

$$
k(t) \asymp \frac{1}{w_{0}\left(w^{-1}(1 / t)\right)} .
$$


As before, here $w(x):=\frac{w_{0}(x)}{w_{1}(x)}$, and $w^{-1}(x)$ is the function inverse to $w(x)$.

The following statement is proved much as Theorem 4.

Theorem 6. Suppose $w_{0}(x)$ and $w_{1}(x)$ satisfy (4.10). Let $\alpha, \beta, \alpha_{0}$, and $\beta_{\infty}$ be the dilation indices of the function $k_{5}(t)=\frac{1}{w_{0}\left(w^{-1}(1 / t)\right)}$, and let $\beta_{\infty} \leq \alpha_{0}$. Then the relation

$$
\left(L_{1}\left(w_{0}\right) \cap \operatorname{Ker} \varphi, L_{1}\left(w_{1}\right) \cap \operatorname{Ker} \varphi\right)_{\theta, q}=\left(L_{1}\left(w_{0}\right), L_{1}\left(w_{1}\right)\right)_{\theta, q} \cap \operatorname{Ker} \varphi
$$

is equivalent to (2.1).

From the preceding theorem we deduce an interesting consequence. Let $a, b, c, d$ be four numbers satisfying $0 \leq a \leq \min (b, c) \leq \max (b, c) \leq d \leq 1$. Then there exists a concave function $\nu(x)$ on $(0, \infty)$ whose dilation indices $\alpha, \beta_{\infty}, \alpha_{0}$, and $\beta$ coincide with $a, b, c$, and $d$.

In order to exhibit such a function, we take a two-sided monotone increasing sequence $\left\{x_{k}\right\}_{k=-\infty}^{\infty}$ of positive numbers such that $x_{0}=1$ and $\lim _{|k| \rightarrow \infty} \frac{x_{k+1}}{x_{k}}=\infty$.

If $b>0$, we define a function $\nu^{\prime}$ in the following way:

$$
\begin{aligned}
& \nu^{\prime}(x)=\left(\frac{x}{x_{2 k}}\right)^{a} \nu^{\prime}\left(x_{2 k}\right), \quad x_{2 k} \leq x \leq x_{2 k+1}, \\
& \nu^{\prime}(x)=\left(\frac{x}{x_{2 k+1}}\right)^{b} \nu^{\prime}\left(x_{2 k+1}\right), \quad x_{2 k+1} \leq x \leq x_{2 k+2},
\end{aligned}
$$

for $k=0,1,2, \ldots$, and

$$
\begin{aligned}
& \nu^{\prime}(x)=\left(\frac{x}{x_{2 k}}\right)^{c} \nu^{\prime}\left(x_{2 k}\right), \quad x_{2 k-1} \leq x \leq x_{2 k}, \\
& \nu^{\prime}(x)=\left(\frac{x}{x_{2 k-1}}\right)^{d} \nu^{\prime}\left(x_{2 k-1}\right), \quad x_{2 k-2} \leq x \leq x_{2 k-1},
\end{aligned}
$$

for $k=0,-1,-2, \ldots$.

If $b=0$ and $d>1$, we define $\nu^{\prime}(x)$ as before if $x \in(0,1]$, and for $x \in[1, \infty)$ we put $\nu^{\prime}(x)=\ln e x$.

Finally, if $d=0$, we put $\nu^{\prime}(x)=\frac{1}{\ln e / x}$ for $x \in(0,1]$ and $\nu^{\prime}(x)=\ln$ ex for $x \in[1, \infty]$.

It can easily be shown that $\nu^{\prime}(x)$ is monotone increasing, $\nu^{\prime}(x) / x$ is monotone decreasing, and the range of $\nu^{\prime}(x)$ is $(0, \infty)$. There exists a monotone increasing concave function $\nu(x)$ such that $\nu^{\prime}(x) \leq \nu(x) \leq 2 \nu^{\prime}(x)(x>0)$; see [17, Theorem 2.1.1]. Clearly, the range of $\nu(x)$ also coincides with $(0, \infty)$. The definition of $\nu^{\prime}$ readily implies that its dilation indices $\alpha, \beta_{\infty}, \alpha_{0}$, and $\beta$ (therefore, also those of $\nu$ ) coincide with $a, b, c$, and $d$, respectively.

We denote $w_{0}(x)=x$ and $w_{1}(x)=x \nu^{-1}(1 / x)\left(\nu^{-1}\right.$ is the inverse function to $\left.\nu\right)$. Since these functions satisfy (4.10) and

$$
\nu(x)=\frac{1}{w_{0}\left(w^{-1}(1 / t)\right)},
$$

an application of Theorem 6 leads to the following result.

Corollary 7. For arbitrary numbers $0 \leq a \leq b \leq c \leq d \leq 1$, there exist weight functions $w_{0}(x)$ and $w_{1}(x)$ such that (4.11) is fulfilled if and only if $\theta \in(0, a) \cup(b, c) \cup(d, 1)$.

Remark 3. The last statement shows that all situations indicated in Theorem 1 are realized indeed, already in the framework of weighted $L_{1}$-spaces. Partial results of the same nature were known earlier; see [1, Corollary 2].

The case of power-type weight functions leads to the most transparent results. If $w_{0}(x)=x^{l}$ and $w_{1}(x)=x^{m}$, it can easily be seen that $(*)$ is fulfilled if $(\mathrm{a}) l>p_{0}-1$ and $m \leq 0$ or (b) $l>p_{0}-1, l>m$, and $0 \leq m<p_{1}-1$. 
By (4.2) and (4.7), we have

$$
v(x)=x^{\frac{p_{1} l-p_{0} m}{p_{1}-p_{0}}}, \quad s(t) \asymp t^{-\frac{r\left(p_{1}-p_{0}\right)}{p_{1} l-p_{0} m+p_{1}-p_{0}}}, \quad k_{2}(t) \asymp t^{\theta_{0}},
$$

where $\theta_{0}=\frac{p_{1}\left(l-p_{0}+1\right)}{p_{1}(l+1)-p_{0}(m+1)}$. Consequently, $\alpha=\beta=\alpha_{0}=\beta_{\infty}=\theta_{0}$, and the preceding results yield the following.

Corollary 8. Suppose $p_{0}, p_{1}\left(1 \leq p_{0} \leq p_{1}<\infty\right), l$, and $m$ satisfy (a) or (b). Then

$$
\left(L_{p_{0}}\left(x^{l}\right) \cap \operatorname{Ker} \varphi, L_{p_{1}}\left(x^{m}\right) \cap \operatorname{Ker} \varphi\right)_{\theta, q}=\left(L_{p_{0}}\left(x^{l}\right), L_{p_{1}}\left(x^{m}\right)\right)_{\theta, q} \cap \operatorname{Ker} \varphi
$$

if and only if $\theta \neq \frac{p_{1}\left(l-p_{0}+1\right)}{p_{1}(l+1)-p_{0}(m+1)}$.

Remark 4. For $p_{0}=p_{1}=q$, the last statement was proved in [1, Corollary 1] by a different method.

Now, we apply the results of $\S 3$ to the evaluation of the $\mathcal{K}$-functional on the couple of intersections $\left(L_{p}\left(w_{0}\right) \cap \operatorname{Ker} \varphi_{g}, L_{p}\left(w_{1}\right) \cap \operatorname{Ker} \varphi_{g}\right)$ for weighted $L_{p}$-spaces, where $\varphi_{g}(f)=$ $\int_{0}^{\infty} f(x) g(x) d x$. Consider two cases.

a) $p_{0}=p_{1}=p>1$. As before, the condition $\varphi_{g} \in\left(L_{p}\left(w_{0}\right) \cap L_{p}\left(w_{1}\right)\right)^{*}$ is equivalent to the condition

$$
g \in L_{p^{\prime}}\left(w_{0}^{-1 /(p-1)}\right)+L_{p^{\prime}}\left(w_{1}^{-1 /(p-1)}\right)=L_{p^{\prime}}\left(\max \left(w_{0}, w_{1}\right)^{-1 /(p-1)}\right) .
$$

Then, as was mentioned already in [2, Subsection 5.4],

$$
\begin{gathered}
\mathcal{K}\left(t^{-1}, \varphi_{g} ; L_{p}\left(w_{0}\right)^{*}, L_{p}\left(w_{1}\right)^{*}\right)=\mathcal{K}\left(t^{-1}, g ; L_{p^{\prime}}\left(w_{0}^{-1 /(p-1)}\right), L_{p^{\prime}}\left(w_{1}^{-1 /(p-1)}\right)\right) \\
\asymp\left(\int_{0}^{\infty}|g(x)|^{p^{\prime}} \min \left(w_{0}^{-1 /(p-1)}(x), t^{-p^{\prime}} w_{1}^{-1 /(p-1)}(x)\right) d x\right)^{1 / p^{\prime}},
\end{gathered}
$$

and the latter function is finite for all $t>0$. In a similar way, we obtain

$$
\mathcal{K}\left(t, f ; L_{p}\left(w_{0}\right), L_{p}\left(w_{1}\right)\right) \asymp\left(\int_{0}^{\infty}|f(x)|^{p} \min \left(w_{0}(x), t^{p} w_{1}(x)\right) d x\right)^{1 / p} .
$$

Therefore, under the assumption

$$
\text { the function } w(x):=\frac{w_{0}(x)}{w_{1}(x)} \text { is monotonic and } \lim _{t \rightarrow 0} w(x)=\lim _{t \rightarrow \infty} \frac{1}{w(x)}=0,
$$

we have

$$
\begin{aligned}
\mathcal{K}(t, f & \left.; L_{p}\left(w_{0}\right), L_{p}\left(w_{1}\right)\right) \\
& \asymp\left(\int_{0}^{w^{-1}\left(t^{p}\right)}|f(x)|^{p} w_{0}(x) d x+t^{p} \int_{w^{-1}\left(t^{p}\right)}^{\infty}|f(x)|^{p} w_{1}(x) d x\right)^{1 / p} \\
& =\left(\left\|f_{0}^{t}\right\|_{L_{p}\left(w_{0}\right)}^{p}+t^{p}\left\|f_{1}^{t}\right\|_{L_{p}\left(w_{1}\right)}^{p}\right)^{1 / p} \\
& \asymp\left\|f_{0}^{t}\right\|_{L_{p}\left(w_{0}\right)}+t\left\|f_{1}^{t}\right\|_{L_{p}\left(w_{1}\right)},
\end{aligned}
$$

where $f_{0}^{t}(x)=f(x) \chi_{\left(0, w^{-1}\left(t^{p}\right)\right)}(x)$ and $f_{1}^{t}(x)=f(x) \chi_{\left(w^{-1}\left(t^{p}\right), \infty\right)}(x)$. The last display shows that the decomposition $f=f_{0}^{t}+f_{1}^{t}$ is optimal (see (3.2)). Moreover, if $f \in$ $L_{p}\left(w_{0}\right) \cap \operatorname{Ker} \varphi_{g}+L_{p}\left(w_{1}\right) \cap \operatorname{Ker} \varphi_{g}$, then $f_{0}^{t}$ belongs to the domain $D_{g}$ of $\varphi_{g}$ for every $t>0$. Thus, Theorem 3 implies the following result. 
Theorem 7. Suppose $p \in(1, \infty)$, the function $w(x)=\frac{w_{0}(x)}{w_{1}(x)}$ satisfies (4.12), and $g \in$ $L_{p^{\prime}}\left(\max \left(w_{0}, w_{1}\right)^{-1 /(p-1)}\right)$. Then

$$
\begin{aligned}
\mathcal{K}(t, f ; & \left.L_{p}\left(w_{0}\right) \cap \operatorname{Ker} \varphi_{g}, L_{p}\left(w_{1}\right) \cap \operatorname{Ker} \varphi_{g}\right) \\
& \asymp\left(\int_{0}^{\infty}|f(x)|^{p} \min \left(w_{0}(x), t^{p} w_{1}(x)\right) d x\right)^{1 / p}+\frac{\left|\int_{0}^{w^{-1}\left(t^{p}\right)} g(x) f(x) d x\right|}{G_{p}(t)},
\end{aligned}
$$

where

$$
G_{p}(t)=\left(\int_{0}^{\infty}|g(x)|^{p^{\prime}} \min \left(w_{0}^{-1 /(p-1)}(x), t^{-p^{\prime}} w_{1}^{-1 /(p-1)}(x)\right) d x\right)^{1 / p^{\prime}} .
$$

If $g(x) \equiv 1$, the formula for the $\mathcal{K}$-functional in Theorem 7 simplifies under certain conditions on $p \in(1, \infty)$ and the weight functions $w_{0}(x)$ and $w_{1}(x)$. Specifically, suppose that

$(* * *) w_{0}(x)$ is monotone increasing and

$$
\int_{x}^{\infty} w_{0}(s)^{\frac{1}{1-p}} d s \leq C_{1} x w_{0}(x)^{\frac{1}{1-p}} \quad(x>0) ;
$$

next, either $w_{1}(x)$ is monotone decreasing and $w_{1}(x) \leq C_{2} w_{1}(2 x)(x>0)$, or both $w_{1}(x)$ and $w(x)=\frac{w_{0}(x)}{w_{1}(x)}$ are monotone increasing, and

$$
\int_{0}^{x} w_{1}(s)^{\frac{1}{1-p}} d s \leq C_{3} x w_{1}(x)^{\frac{1}{1-p}} \quad(x>0) .
$$

Arguing as we did before Corollary 6, we easily obtain the following result, which was established in [1, Theorem 3] by a different method, namely, on the basis of direct estimates.

Corollary 9. Suppose $p \in(1, \infty), w_{0}(x)$, and $w_{1}(x)$ satisfy $(* * *)$. Then

$$
\begin{aligned}
\mathcal{K}(t, f ; & \left.L_{p}\left(w_{0}\right) \cap \operatorname{Ker} \varphi, L_{p}\left(w_{1}\right) \cap \operatorname{Ker} \varphi\right) \\
\asymp & \left(\int_{0}^{\infty}|f(x)|^{p} \min \left(w_{0}(x), t^{p} w_{1}(x)\right) d x\right)^{1 / p} \\
& +\left(w^{-1}\left(t^{p}\right)\right)^{1 / p-1} w_{0}^{1 / p}\left(w^{-1}\left(t^{p}\right)\right)\left|\int_{0}^{w^{-1}\left(t^{p}\right)} f(x) d x\right| .
\end{aligned}
$$

b) $p_{0}=p_{1}=1$. In this case the condition $\varphi_{g} \in\left(L_{1}\left(w_{0}\right) \cap L_{1}\left(w_{1}\right)\right)^{*}$ emerges, which is equivalent to

$$
g \in L_{\infty}\left(1 / w_{0}\right)+L_{\infty}\left(1 / w_{1}\right)=L_{\infty}\left(1 / \max \left(w_{0}, w_{1}\right)\right) .
$$

Now, for all $t>0$ the following function is finite:

$$
\begin{aligned}
\mathcal{K}\left(t, \varphi_{g} ; L_{1}\left(w_{0}\right)^{*}, L_{1}\left(w_{1}\right)^{*}\right) & =\mathcal{K}\left(t, g ; L_{\infty}\left(1 / w_{0}\right), L_{\infty}\left(1 / w_{1}\right)\right) \\
& =\sup _{x>0}\left(|g(x)| \min \left(\frac{1}{w_{0}(x)}, \frac{t}{w_{1}(x)}\right)\right) .
\end{aligned}
$$

As in the case of $p>1$, if (4.12) is fulfilled, then

$$
\mathcal{K}\left(t, f ; L_{1}\left(w_{0}\right), L_{1}\left(w_{1}\right)\right)=\left\|f_{0}^{t}\right\|_{L_{1}\left(w_{0}\right)}+t\left\|f_{1}^{t}\right\|_{L_{1}\left(w_{1}\right)},
$$

where $f_{0}^{t}(x)=f(x) \chi_{\left(0, w^{-1}(t)\right)}(x)$ and $f_{1}^{t}(x)=f(x) \chi_{\left(w^{-1}(t), \infty\right)}(x)$. Thus, the decomposition $f=f_{0}^{t}+f_{1}^{t}$ is optimal. Moreover, if $f \in L_{1}\left(w_{0}\right) \cap \operatorname{Ker} \varphi_{g}+L_{1}\left(w_{1}\right) \cap \operatorname{Ker} \varphi_{g}$, then $f_{0}^{t} \in D_{g}$ for every $t>0$. Therefore, Theorem 3 yields yet another theorem. 
Theorem 8. If (4.12) is fulfilled and $g \in L_{\infty}\left(1 / \max \left(w_{0}, w_{1}\right)\right)$, then

$$
\begin{aligned}
\mathcal{K}(t, f & \left.; L_{1}\left(w_{0}\right) \cap \operatorname{Ker} \varphi_{g}, L_{1}\left(w_{1}\right) \cap \operatorname{Ker} \varphi_{g}\right) \\
& \asymp\left(\int_{0}^{\infty}|f(x)| \min \left(w_{0}(x), t w_{1}(x)\right) d x\right)+\frac{\left|\int_{0}^{w^{-1}(t)} g(x) f(x) d x\right|}{G_{1}(t)},
\end{aligned}
$$

where

$$
G_{1}(t)=\sup _{x>0}\left(|g(x)| \min \left(\frac{1}{w_{0}(x)}, \frac{1}{t w_{1}(x)}\right)\right) .
$$

If $g(x) \equiv 1$ and the weight functions are sufficiently regular, the formula for the $\mathcal{K}$-functional on the couple of intersections simplifies somewhat.

Corollary 10. If $w_{0}(x)$ and $w_{1}(x)$ satisfy (4.10), then

$$
\begin{aligned}
\mathcal{K}(t, f ; & \left.L_{1}\left(w_{0}\right) \cap \operatorname{Ker} \varphi, L_{1}\left(w_{1}\right) \cap \operatorname{Ker} \varphi\right) \\
& \asymp\left(\int_{0}^{\infty}|f(x)| \min \left(w_{0}(x), t w_{1}(x)\right) d x\right)+w_{0}\left(w^{-1}(t)\right)\left|\int_{0}^{w^{-1}(t)} f(x) d x\right| .
\end{aligned}
$$

Remark 5. Under the additional assumption $w_{1}(x / 2) \leq A w_{1}(x)(x>0)$, Corollary 10 was proved by a different method in [1] (see Theorem 3 ).

The author is grateful to Prof. L. Maligranda for a useful discussion of certain issues related to the proof of Theorem 2 .

\section{REFERENCES}

[1] N. Krugljak, L. Maligranda, and L.-E. Persson, The failure of the Hardy inequality and interpolation of intersections, Ark. Mat. 37 (1999), 323-344. MR.1714765 (2000h:46035)

[2] J. Bergh and J. Löfström, Interpolation spaces. An introduction, Grundlehren Math. Wiss., vol. 223, Springer-Verlag, Berlin-New York, 1976. MR0482275 (58:2349)

[3] S. Ivanov and N. Kalton, Interpolation of subspaces and applications to exponential bases, Algebra $\mathrm{i}$ Analiz 13 (2001), no. 2, 93-115; English transl., St. Petersburg Math. J. 13 (2002), no. 2, 221-239. MR 1834861 (2002f:46147)

[4] J.-L. Lions and E. Magenes, Problèmes aux limites non homogènes et applications. Vols. 1-2, Dunod, Paris, 1968. MR0247243(40:512) MR0247244(40:513)

[5] H. Triebel, Eine Bemerkung zur nicht-kommutativen Interpolation, Math. Nachr. 69 (1975), 57-60. MR0390797 (52:11620)

[6] - Interpolation theory, function spaces, differential operators, North-Holland Math. Library, vol. 18, North-Holland Publ. Co., Amsterdam-New York, 1978. MR0503903 (80i:46032b)

[7] L. Maligranda, On commutativity of interpolation with intersection, Rend. Circ. Mat. Palermo (2) Suppl. No. 10 (1985), 113-118 (1986). MR0894276 (88j:46067)

[8] R. Wallsten, Remarks on interpolation of subspaces, Function Spaces and Applications (Lund, 1986), Lecture Notes in Math., vol. 1302, Springer, Berlin, 1988, pp. 410-419. MR0942285 (89h:46042)

[9] G. Pisier, Interpolation between $H^{p}$ spaces and noncommutative generalizations. I, Pacific J. Math. 155 (1992), 341-368. MR.1178030 (93f:46111)

[10] S. Janson, Interpolation of subcouples and quotient couples, Ark. Mat. 31 (1993), 307-338. MR.1263557(95c:46124)

[11] S. V. Kislyakov and Q. Xu, Real interpolation and singular integrals, Algebra i Analiz 8 (1996), no. 4, 75-109; English transl., St. Petersburg Math. J. 8 (1997), no. 4, 593-615. MR1418256 (98c:46161)

[12] J. Löfström, Interpolation of subspaces, Techn. Rep. no. 10, Univ. of Göteborg, 1997, 63 pp.

[13] S. V. Astashkin, About interpolation of subspaces of rearrangement invariant spaces generated by Rademacher system, Int. J. Math. Math. Sci. 25 (2001), no. 7, 451-465. MR.1823608 (2002e:46029)

[14] S. Kaijser and P. Sunehag, Interpolation of subspaces and the unit problem, Function Spaces, Interpolation Theory and Related Topics (Lund, 2000), de Gruyter, Berlin, 2002, pp. 345-353. MR.1943292 (2003k:46106)

[15] S. V. Astashkin, E. M. Semenov, and F. A. Sukochev, The Banach-Saks p-property, Math. Ann. 332 (2005), 879-900. MR 2179781 
[16] Yu. A. Brudnyi and N. Ya. Krugljak, Interpolation functors and interpolation spaces. Vol. 1, North-Holland Math. Library, vol. 47, North-Holland Publ. Co., Amsterdam, 1991. MR1107298 (93b:46141)

[17] S. G. Kreĭn, Yu. I. Petunin, and E. M. Semenov, Interpolation of linear operators, "Nauka", Moscow, 1978; English transl., Transl. Math. Monogr., vol. 54, Amer. Math. Soc., Providence, RI, 1982. MR0506343(81f:46086) MR0649411 (84j:46103)

Samara State University, Ul. Akademika Pavlova 1, Samara 443011, Russia

E-mail address: astashkn@ssu.samara.ru

Received 30/SEP/2003

Translated by S. V. KISLYAKOV 